\title{
评述
}

\section{钛硅分子篮催化剂高效钛位点的理性构筑与调控 及催化烯烃环氧化的性能}

\author{
林栋 $^{1}$, 冯翔 ${ }^{*}$, 刘熠斌 ${ }^{1}$, Chen $\mathrm{De}^{1,2}$, 杨朝合 ${ }^{1}$ \\ 1. 中国石油大学(华东)重质油国家重点实验室, 青岛 266580 \\ 2. 挪威科技大学化学工程系, 特隆赫姆 7491 \\ *通讯作者, E-mail: xiangfeng@upc.edu.cn
}

收稿日期: 2021-08-16; 接受日期: 2021-10-13; 网络版发表日期: 2022-01-21

国家自然科学基金(编号: 21978325, 21776312, 22078364)和山东省自然科学基金(编号: ZR2020KB006)资助项目

\begin{abstract}
摘要随着化工产品高端化战略的不断推进, 以 TS-1 为代表的钛硅分子篎催化剂由于具备独特的选择性氧化 能力而引起了广泛关注, 目前已经成功应用于烯烃环氧化、环已酮氨氧化、苯酚羟基化等领域. TS- 1 型钛硅分子 篎也因此被誉为分子篮催化领域的里程碑. 理性设计与精准构筑TS-1中高性能钛位点是高效催化烯烃环氧化的 关键. 本文首先综述了构筑高性能TS-1钛位点的三大策略, 包括在原子尺度上设计钛位点的配位结构、在界面上 调节钛位点的微环境, 以及在纳米颗粒中改善钛位点的可接触性. 此外, 详细阐述了上述不同策略对烯烃环氧化 的影响机制及相应的构效关系, 总结了不同策略下的构筑方法和表征手段, 并分析了高性能钛硅分子篎当前面临 的挑战与未来的发展方向.
\end{abstract}

关键词钛硅分子䇻, TS-1, 烯烃环氧化, 高效钛位点, 构筑策略

\section{1 引言}

面对环境逐步恶化与资源加速短缺的双重挑战, 能源化工领域开始聚焦于精准催化, 力争实现“原料多 元化、过程绿色化、产品高端化”的行业发展目 标 ${ }^{[1 \sim 4]}$. 新的战略目标要求清洁高效地利用化石能源, 加速推动其下游相关高附加值产品的综合利用 ${ }^{[5,6]}$. 因 此, 以炼化一体化带动烯烃下游高端化学品合成, 打造 高端化工产业新亮点、新优势是当前炼化领域的迫切 需求 ${ }^{[7,8]}$. 其中, 烯烃下游的高附加值环氧化学品作为
一种重要的有机化工中间体，被广泛应用于医药、汽 车、食品、农业和建筑等领域 ${ }^{[911]}$. 然而, 目前除乙 烯、丙烯外，部分烯烃环氧化生产采用有机过氧酸 法、氯醇法等环境污染严重或者加工过程复杂、联产 物繁多的工艺流程 ${ }^{[12 \sim 15]}$, 亟需发展新型绿色工艺. 因 此，大力发展绿色环氧化工艺符合绿色化工可持续发 展的战略需求与目标.

以TS-1 为代表的钛硅分子篮与过氧化氢组成的环 氧化催化体系由于其绿色环保的特性受到广泛关 注 $^{[16-22]}$, 该体系已经被广泛应用于一系列反应中 ${ }^{[23 \sim 26]}$,

引用格式: Lin D, Feng X, Liu Y, Chen D, Yang C. Rationally constructed and regulated highly efficient Ti sites of titanium silicalite for olefin epoxidation. Sci Sin Chim, 2022, 52: 560-579, doi: 10.1360/SSC-2021-0182 
如烯烃环氧化、芳烃的羟基化、环己酮的氨氧化、烷 烃氧化、氧化脱硫以及醇和胺的氧化等(图 1 所 示 ${ }^{[27 \sim 29]}$. 此外, 该催化体系反应过程简单、可操作性 强; 反应条件温和, 反应温度一般不高于 $100^{\circ} \mathrm{C}$, 反应压 力多为常压, 安全性能得到极大改善; 采用过氧化氢做 氧化剂，产物选择性高、环境污染较小 ${ }^{[30 \sim 32]}$. 在上述 体系中，钛硅分子篎中独特的选择性氧化活性位为骨 架中的钛位点 ${ }^{[33 ~ 36]}$, 因此, 精准构筑环氧化钛活性位 点成为理性设计高效TS-1催化剂的关键.

随着环境保护政策的加速推进，绿色化学理念的 深入人心，本文以烯烃绿色环氧化新过程为反应实例， 在不同层次上总结概括钛硅分子篮TS-1催化剂高效钛 位点的设计策略，详细阐述了不同策略对烯烃环氧化 的影响机制与反应机理，并总结了相应的构筑方法和 表征手段. 针对原子尺度上的活性中心钛位点的配位 形式、界面上的微反应环境以及纳米颗粒中的可接触 性三个方面，分别提出通过构造不同配位形式的钛位 点、设计分子篮钛位点周围的微反应环境，以及改善 烯烃反应物和相关产物的传质扩散三种方法来合成高 性能钛位点. 本文有关高效钛位点的构筑策略, 将有助 于推动基于钛硅分子篮的烯烃环氧化工业化进程.

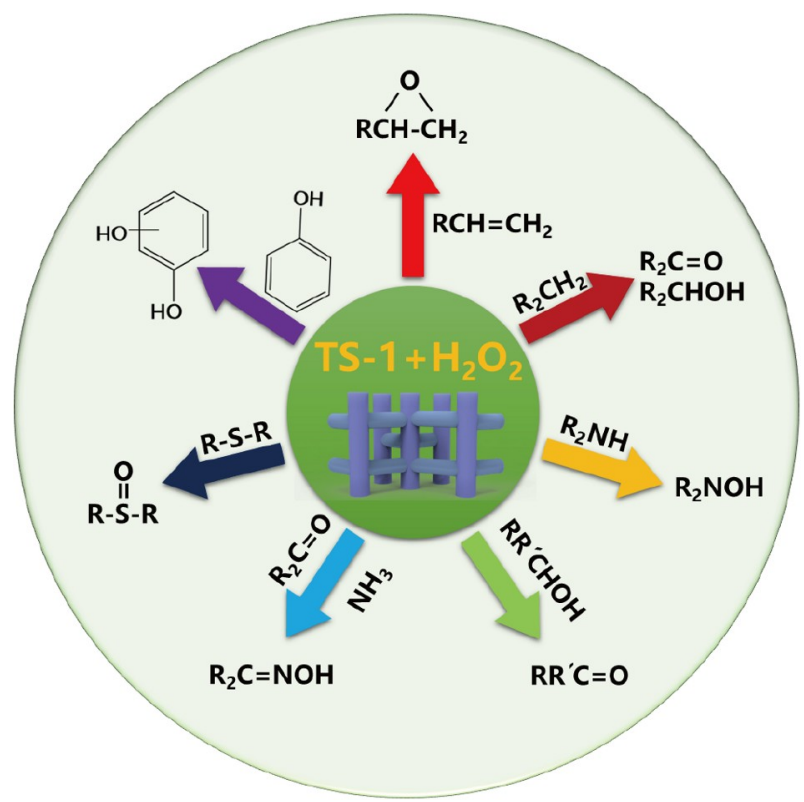

图 $1 \mathrm{TS}-1$ 在不同反应中的应用(网络版彩图)

Figure 1 The applications of TS-1 in different reactions (color online).

\section{2 原子尺度钛位点配位形式}

由于钛硅分子篎中的骨架钛物种通常被认为是烯 烃环氧化的活性中心 ${ }^{[37 \sim 39]}$, 因此直接从源头设计和调 控骨架钛物种的配位形式对于合成高性能烯烃环氧化 TS-1催化剂极其重要. 然而, 在原子尺度上精准合成具 有特定配位形式的骨架钛物种仍然具有挑战性. 目前, 通常认为非骨架钛物种(具有八面体配位形式的钛物 种)和锐钛矿形式的钛物种(二氧化钛)不利于选择性氧 化反应 ${ }^{[40-43]}$, 并且锐钛矿二氧化钛会加速氧化试剂过 氧化氢 $\left(\mathrm{H}_{2} \mathrm{O}_{2}\right)$ 分解 ${ }^{[44,45]}$, 降低反应的活性. 然而, 当前 不同体系中烯烃环氧化的活性钛物种结构仍然没有统 一的定论, 具有争议的烯烃环氧化活性钛位点主要包 括孤立的四配位骨架钛 $\left(\mathrm{TiO}_{4}\right)^{[46]}$ 、五配位骨架钛 $\left(\mathrm{TiO}_{5}\right)^{[47]}$ 和单核形式的六配位骨架钛 $\left(\mathrm{TiO}_{6}\right)^{[39]}$ (图2).

\section{1 孤立的四配位骨架钛}

目前，研究者最广泛认可的烯烃环氧化活性中心 为孤立的四配位骨架铁 ${ }^{[43,48]}$. 过氧化氢通过与孤立的 四配位骨架铁相互作用形成Ti-OOH中间体, 然后烯烃 与 Ti-OOH发生环氧化反应生成相关的环氧化产物 ${ }^{[49]}$. 然而，制备只含孤立四配位骨架钛TS-1的条件较为苛 刻. 自发条件下，由于硅前驱体的水解速率要远远慢 于钛前驱体的水解速率，这种水解速率的不匹配极易 生成锐钛矿型二氧化钛 ${ }^{[50,51]}$. 因此，制备富含孤立四 配位骨架钛物种的方法主要是通过匹配硅和钛前驱体 物种的水解速率而实现的，如通过在前驱体溶液中添 加一系列添加剂(吐温-20、异丙醇、碳酸铵、过氧化 氢、Triton X-100)和严格控制钛源滴加速率来降低钛 前驱体水解速率 ${ }^{[50,52 \sim 56]}$.

本研究团队 ${ }^{[57]}$ 提出调控前驱体水解速率匹配的 “逆向聚合法”，即直接将钛源、硅源和模板剂混合先 形成钛低聚体, 进而通过紫外光原位引入的羟基自由
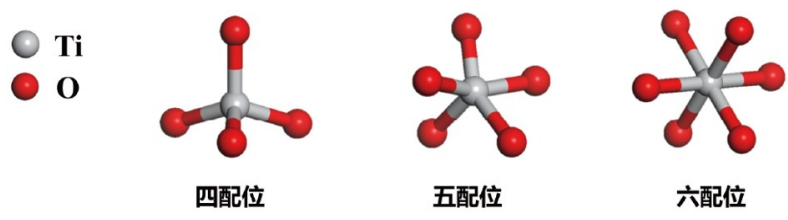

图 2 TS-1中骨架钛物种的不同配位形式(网络版彩图)

Figure 2 The different coordination modes of $\mathrm{Ti}$ species in the framework of TS-1 (color online). 
基来同时解聚钛低聚体为钛单体，并加速硅前驱体水 解，最终实现钛与硅前驱体水解速率匹配(图3a). 紫 外-可见光(UV-Vis)谱图和密度泛函理论(DFT)计算表 明(图3b，c)，通过紫外线辐射原位产生的羟基自由基 可以有效解聚钛低聚体，成功保证了在无须添加添加 剂的情况下实现硅钛分子篮快速合成，并有效避免了 非骨架钛 $\left(\mathrm{TiO}_{2}\right)$ 物种的生成，促使TS-1中钛物种以四 配位骨架钛的形式存在. 相比于传统的水热合成方法, 该方法具有明显的特点: (1) 绿色环保, 不需要多种添 加剂, 如表面活性剂(Tween-20)和溶剂(异丙醇). (2) 合 成方法简便、高效. 传统方法中缓慢的硅源水解速率 和严格控制的钣源滴加速率导致通常需要至少 $6 \mathrm{~h}$ 来 制备前驱体溶液，此外高温蒸发除掉溶液中的醇类至 少需要 $2 \mathrm{~h}$. 然而“逆向聚合法”只需要直接将钛源、硅 源和模板剂直接混合并在紫外光下辐射 $1 \mathrm{~h}$ 即可进行
晶化, 操作难度大大降低、操作过程极大简化, 更有利 于钛硅分子篮合成的工业放大. (3) 分子篮收率比传统 水热法高约 $10 \%$. (4) 分子篮外表面孤立的四配位骨架 钛含量更低, 硅含量更高. 更高的表面硅含量可以有效 提高外表面催化惰性，进而通过构筑惰性外表面来有 效抑制环氧化合物在扩散过程中造成的分解. (5) 具 有更小的颗粒粒径和更大的分子篮比表面积. (6) 具 有普适性, 同时适用于其他含钛分子篮的合成, 如TS1、TS-2和Ti- $\beta$, 此外还适用于含Zr等易水解物种的分 子篮合成. “逆向聚合法”为钛硅分子篎的工业合成提 供了一种提质增效的全新思路.

除了可以通过调控前驱体的水解速率来改变孤立 四配位骨架钛物种的含量外, Zhang等 ${ }^{[56]}$ 在分子篮前 驱体溶液中添加Triton X-100来降低分子篮的晶化速 率(即降低MFI分子篎结构的生成速率), 同时引入旋转 (a)

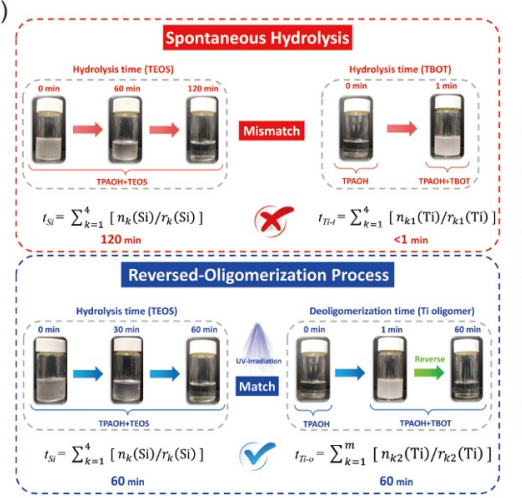

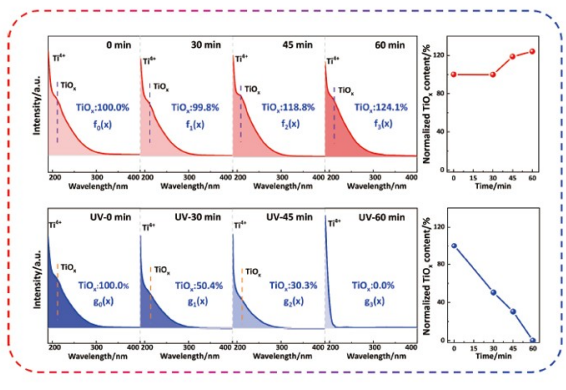

(e)

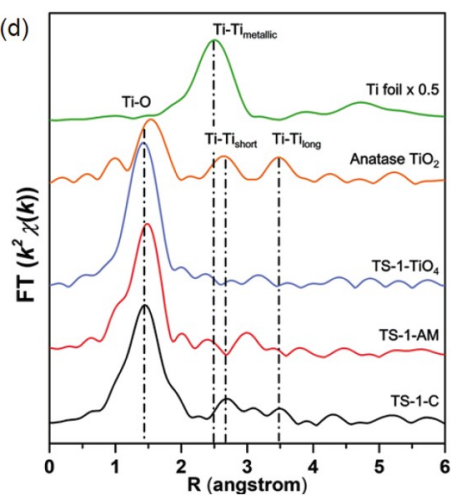

(b)
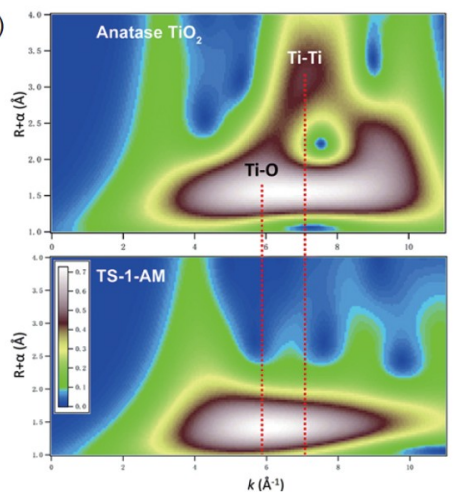

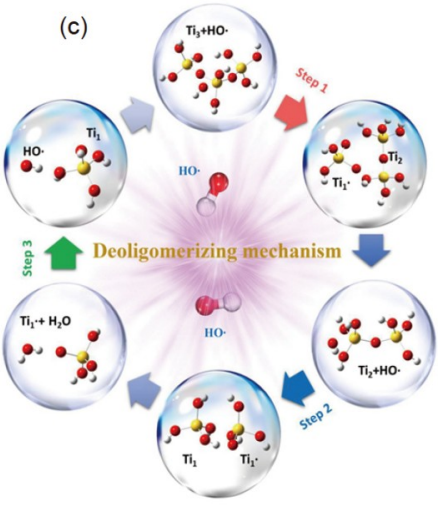

(f)

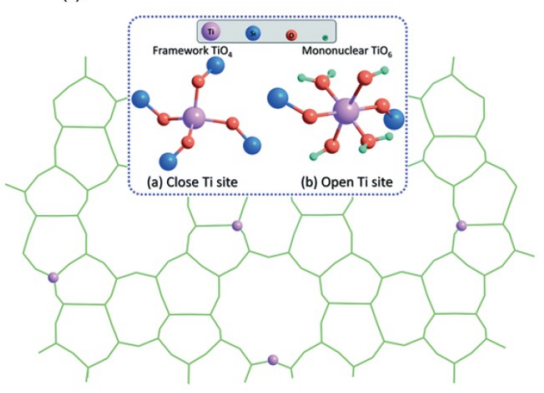

图 3 (a) 紫外条件与常规条件下硅钛物种的水解速率 ${ }^{[57]}$; (b) 紫外条件与常规条件下钛物种的UV-Vis谱图 ${ }^{[57]}$; (c) 羟基自由基 解聚钛低聚物路径图 ${ }^{[57]}$; (d) 不同TS-1样品的EXAFS谱图 ${ }^{[39]}$; (e) TS-1和 $\mathrm{TiO}_{2}$ 样品的小波变换图 ${ }^{[39]}$; (f) 单核 $\mathrm{TiO}_{6}$ 骨架钛示意 图 ${ }^{[39]}$ (网络版彩图)

Figure 3 (a) The hydrolysis rates of $\mathrm{Si}$ and Ti species in the ultraviolet (UV)-irradiation and spontaneous process [57]; (b) the UV-visible (Vis) spectra of Ti species in the UV-irradiation and spontaneous process [57]; (c) the deoligomerization pathway of Ti oligomers by hydroxyl free radicals

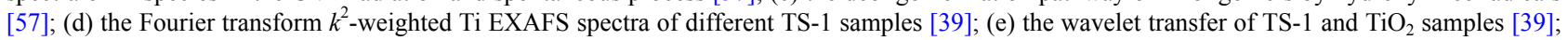
(f) the schematic of mononuclear $\mathrm{TiO}_{6}$ species [39] (color online). 
晶化来改善溶胶中钛物种分散的情况, 从而促进钛物 种与硅物种之间的缩合, 进而加速钛物种在分子篮骨 架中的插入速率，从而有效匹配了硅钛物种在TS- 1 钛 硅分子篮中的相对嵌入速率，进而抑制了非骨架钛 $\left(\mathrm{TiO}_{2}\right)$ 的生成. 相比于传统方法合成的钛硅分子篮，该 方法合成的小尺寸无非骨架钛的钛硅分子篮具有更高 的1-已烯环氧化转化率(52.6\%)和选择性( $97 \%$ ). 这种差 异主要是由于Triton X-100的加入可以作为软模板剂合 成微介复合的多级孔道铁硅分子篮, 从而有效缩短了大 分子烯烃的扩散路径并降低了扩散的空间位阻, 从而有 效提高烯烃环氧化性能. $\mathrm{Ahn}$ 等 ${ }^{[58]}$ 采用微波加热 $\mathrm{SiO}_{2}$ $\mathrm{TiO}_{2}$ 干凝胶和模板剂四丙基氢氧化铵(TPAOH)的混合 物来合成TS-1分子篮. 结果表明, $30 \mathrm{~min}$ 的微波加热即 可促使分子篮收率达到 $90 \%$, 合成时间远低于传统合成 所需要的1 2天. 傅里叶转换红外线光谱(FT-IR)分析和 UV-Vis光谱表明，微波合成的TS-1分子篮中的钛物种 仍然为孤立的四配位骨架铁, 并且在1-已烯环氧化中展 现出与传统催化剂相似的催化活性. 此外, $\mathrm{Xu}$ 等 ${ }^{[59]}$ 通过 微波加热合成含钛的MFI分子篮, UV-vis表明该分子篎 主要为四配位的骨架钛物种. 此外, IR谱图和扫描电子 显微镜(SEM)图片表明，微波加热会促使不同分子篮晶 体上的硅羟基相互缩合, 从而导致分子篎晶体之间相互 堆叠, 进而有效降低载体疏水性. 苯乙烯环氧化反应表 明，微波合成的含钛分子篮具有更高的疏水性，从而呈 现出更高的环氧化反应选择性.

除了以上调控硅源和钛源水解速率的方法外，不 同种类的钛源也会影响钛源水解速率，进而影响钛硅 分子篮中的钛物种. Wang 等 ${ }^{[60}$ 研究了不同钛源 $\left(\mathrm{TiCl}_{3}\right.$ 、钛酸四丁酯 $(\mathrm{TBOT})$ 和 $\left.\mathrm{Ti}\left(\mathrm{SO}_{4}\right)_{2}\right)$ 的水解速率和 硅源水解速率的匹配关系. 在以 $\mathrm{TPABr}$ 为模板剂、氨 水为碱源时, 溶液的 $\mathrm{pH}$ 为 $10.5 \sim 11.5$, 而 $\mathrm{TBOT}$ 和 $\mathrm{TiCl}_{3}$ 的水解则需要更高的碱度 $(\mathrm{pH}$ 为 $12.0 \sim 13.5)$ 来匹配硅源 的水解. 因此在该体系下, 以 $\mathrm{Ti}\left(\mathrm{SO}_{4}\right)_{2}$ 为钛源, 能够减少 非骨架钛的生成, 提高骨架钛含量, 并且在氯丙烯环氧 化中展现出最高的环氧化产物收率(99.04\%). Liu等 ${ }^{[61]}$ 分别采用 $\mathrm{TiCl}_{3} 、 \mathrm{TBOT} 、 \mathrm{Ti}\left(\mathrm{SO}_{4}\right)_{2}$ 作为钛源， $\mathrm{TPABr}$ 作 为模板剂, 乙醇胺为碱源, 气相二氧化硅作为硅源合 成TS-1分子笁. 以 TBOT为钛源的混合溶胶具有更高 的碱度，从而提高了分子篮的相对结晶度以及骨架钛 含量. 以TBOT为钛源合成出的 TS- 1 在丙烯环氧化反 应中呈现出最高的环氧丙烷收率.

\section{2 其他配位形式的骨架钛}

五配位钛物种主要分为两种, 一种是通过孤立的 四配位钛物种转化而来, 其为反应的中间体; 另一种 则是钛原子与五个 $\mathrm{SiO}_{4}$ 四面体中的 $\mathrm{O}$ 原子相互成键而 实现.二者的存在方式明显不同, 反应中间体形成的五 配位结构只有在反应条件下才会产生，而后者则为催 化剂的固有结构. 对于前者, 主要是在添加醇类溶剂 的情况下,醇类中的氧原子会与活性中间体Ti-OOH中 的四配位钛原子配位，从而形成一种五元环的中间体 结构 ${ }^{[62]}$. 烯烃反应物的双键通过亲核反应被这种五元 环结构中的 $\beta$ 位上的氧原子进攻, 从而高效地选择性 氧化烯烃生成相关环氧化产物, 这种五元环机理清晰 地解释了醇类的溶剂效应, 因而得到研究者们的认 可 ${ }^{[63-66]}$. 由于其他配位形式钛物种均为催化剂固有结 构, 因此, 这里主要强调固有结构的五配位钛物种和 其他配位形式的铁物种. 对于后一种五配位钛物种, 其主要是在晶化过程中钛原子与五个 $\mathrm{SiO}_{4}$ 四面体配位 并进一步生长为有序三维MFI孔道得到. Zuo 等 ${ }^{[40]}$ 通过 改变小晶体TS-1合成溶胶中的硅钛比来调控五配位骨 架钛物种(钛原子与五个 $\mathrm{SiO}_{4}$ 四面体配位)的数量. 尽管 $\mathrm{TiO}_{4}$ 与五个 $\mathrm{SiO}_{4}$ 四面体配位形成五配位骨架钣物种, 其钛物种化学环境并不完全一致, 因此五配位骨架钛 物种也被称为具有缺陷的四配位铁物种. UV-Vis、X 射线吸收近边结构(XANES)谱图和反应结果表明, 适 量的硅钛比(如 $\mathrm{Si} / \mathrm{Ti}=80$ ) 会促进五配位骨架钛物种的 生成，进一步将前驱体合成溶胶中的硅钛比从 80 降低 到 50 , 将会提高载体中的八面体配位非骨架钛含量. 其中五配位钛物种是活性中心，而八面体配位钛物种 是惰性物种.

单核六配位钛物种是TS-1 钛硅分子篮骨架中一种 全新的钛物种, 其首次被 $\mathrm{Xu}$ 等 ${ }^{[39]}$ 在晶种和微波的协助 下通过一步快速晶化的方法成功合成. 在XANES光谱 的4960 4970 eV区域内, 相比于纯四配位钛物种, 六配 位钛物种的峰强度降低, 这是由于六配位钛物种是一 种扭曲的钛物种。在4980 4990 eV区域内，六配位钛 物种缺少明显的特征峰, 即缺少长程和中程有序的结 构, 这是由于钋物种在该钛硅分子篮中更加分散. EXAFS光谱则表明，含有六配位钛物种的钛硅分子篮不 含有 $\mathrm{Ti}-\mathrm{Ti}$ 键，表明 $\mathrm{Ti}$ 原子在分子篮中为孤立的状态. UV-Vis、XANES和X射线吸收精细结构(EXAFS)光谱 表明，单核的六配位骨架钛与六个氧原子配位(图3d, 
e), 而这六个氧原子只有部分与TS-1骨架中的硅原子 配位，因此这种六配位的钛位点是一种开放的钛位点 (图3f). 但是这种开放的钛位点在 $550^{\circ} \mathrm{C}$ 下焙烧 $6 \mathrm{~h}$ 仍然 能够稳定存在．在1-已烯环氧化反应中具有单核六配 位骨架钛的钛硅分子篮相比于传统TS-1钛硅分子篮, 转化数(TON)值提高了 $70 \%$ 左右, 这种高已烯环氧化活 性归结于独特的单核六配位骨架铁物种.

2020年，Gordon等 ${ }^{[67]}$ 提出了一个颠覆式的观点， 他们认为TS-1中环氧化的关键位点是一种双核的钛位 点而不是大家广泛认为的孤立的四配位、五配位和六 配位骨架钛位点. 固体核磁 ${ }^{17} \mathrm{O} N \mathrm{NM}$ 谱图表明, 这种双 核钛位点与过氧化氢 $\left(\mathrm{H}_{2} \mathrm{O}_{2}\right)$ 之间的接触作用会促进桥 联过氧化物的形成(图4a), 并且这些桥联过氧化物会
进一步与烯烃反应生成相应的环氧化产物。DFT计算 表明(图4b), 双核钛位点(TS1-O)通过与过氧化氢作用 生成 TS $1-\mathrm{O}_{2}$, 紧接着通过脱去一分子水形成 TS 1 $(\mathrm{OOH})_{2}$, 该中间产物会进一步和丙烯反应生成环氧丙 烷. 因此, 相邻的Ti位点之间的协同作用开辟了一个更 有效的环氧化路径(即氧转移过渡态机理), 为高效钛 硅分子篎的设计提供了一种全新的思路.

要想实现钛活性位的精准构筑, 不仅需要实验上 的合成手段，还依赖于材料上的表征方法. 近来, XANES和EXAFS逐渐成为在原子尺度上研究钛配位形 式的有力手段. 烯烃环氧化的活性物种(如单核钛物种 Ti-OOH和双核钛物种HOOTi-TiOOH) 可以通过原位紫 外可见光光谱和固体核磁 ${ }^{17} \mathrm{O} N \mathrm{NR}$ 谱图来进行表征. 此

(a)
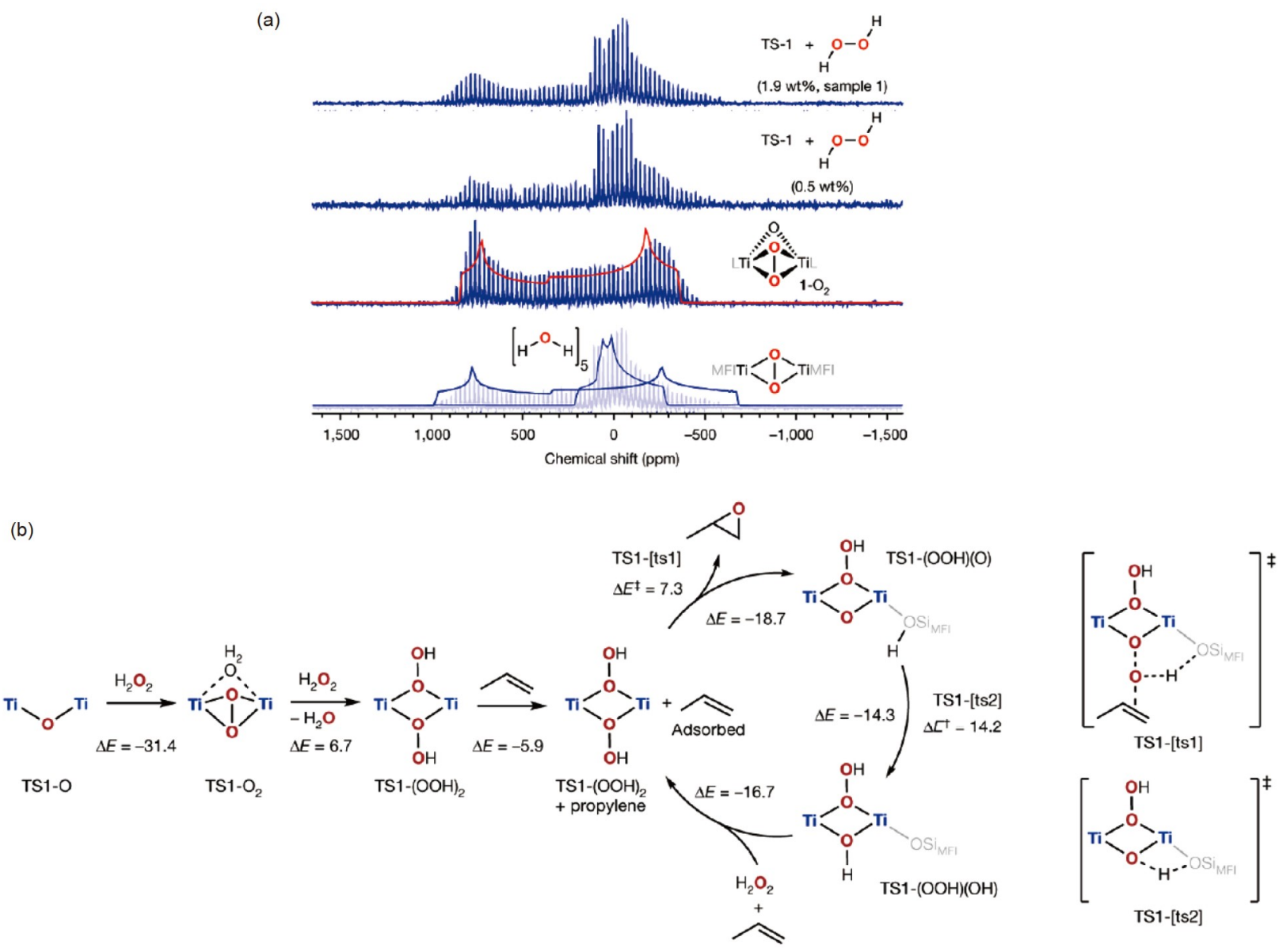

图 4 (a) 实验测定与理论计算的 TS- 1 与 $\mathrm{H}_{2} \mathrm{O}_{2}, \mathrm{O}_{2}, \mathrm{H}_{2} \mathrm{O}$ 接触的不同 ${ }^{17} \mathrm{O} N M R$ 谱图 ${ }^{[67]}$; (b) 双核钛位点上丙烯环氧化的理论计算 势能面(电子能单位为 $\mathrm{kcal} / \mathrm{mol})^{[67]}$ (网络版彩图)

Figure 4 (a) The experimental and calculated solid-state ${ }^{17} \mathrm{O}$ nuclear magnetic resonance $\left({ }^{17} \mathrm{O}\right.$ NMR) spectra of TS-1 contacted with $\mathrm{H}_{2} \mathrm{O}_{2}, \mathrm{O}_{2}$ and $\mathrm{H}_{2} \mathrm{O}$ [67]; (b) the calculated energy surface of propylene epoxidation at the dinuclear Ti site (the unit of electronic energies is $\mathrm{kcal} / \mathrm{mol}$ ) [67] (color online). 
外, DFT计算可以在原子尺度上提供一个新的视角来揭 示和比较不同反应路径的吸附形式和反应能垒等. 不同 形式的骨架钛物种中, $\mathrm{TiO}_{4}$ 物种的构筑策略可以确保 TS-1中只含有四配位骨架钛, 而 $\mathrm{TiO}_{5}$ 和 $\mathrm{TiO}_{6}$ 物种的构筑 策略则使TS-1在包含相应五配位和六配位骨架钛的同 时也包含四配位骨架钛. 总之, 精确设计 TS-1 钛硅分子 笚中钛位点的配位形式具有巨大的挑战性, 但是有望从 钛活性中心的源头上有效提高烯烃环氧化的反应性能.

\section{3 界面微反应环境}

钛硅分子篮的环氧化活性中心为骨架钛物种, 因 此在界面上调节骨架钛物种周边的微反应环境将会深 刻影响环氧化产物接下来的行为(如脱附、开环、低 聚等). 目前, 钛物种的微反应环境影响因素主要包括 钛物种周边的酸碱性位点以及硅羟基, 精准调控二者 的数目以及空间分布将会改善环氧化产物的反应行 为, 从而极大地提高烯烃环氧化反应的活性、选择性 以及稳定性.

\section{1 调控钛物种周边疏水性}

精准调控钛位点周边的硅着基 $(\mathrm{Si}-\mathrm{OH})$ 数目能够 有效改善钛硅分子篎的亲疏水性, 硅羟基数目越少则 钛硅分子篮的疏水性越强, 反之则亲水性越强 ${ }^{[68]}$. 钛 硅分子篮的疏水性会影响环氧化产物的脱附, 更高的 疏水性能够降低环氧化产物开环聚合的可能性, 提高 烯烃环氧化的活性、选择性以及稳定性 ${ }^{[69]}$. 目前, 为 提高钛硅分子篮疏水性而广泛采用的方法包括硅烷化 处理 ${ }^{[70 \sim 72]}$ 和氟化铵 $\left(\mathrm{NH}_{4} \mathrm{~F}\right)$ 处理 ${ }^{[73 \sim 75]}$.

硅烷化处理是通过将硅烷试剂经过缩合反应嫁接 到硅羟基上, 从而将末端的硅羟基官能团替换为硅烷 醇基官能团. 相比于硅羟基, 硅烷醇基基团同环氧化 产物的相互作用更弱, 更有利于环氧化产物的及时脱 附，从而抑制其在钛硅分子篮表面开环聚合并减少活 性位的覆盖 ${ }^{[76]}$. Wang 等 ${ }^{[77]}$ 采用三甲基氯硅烷作为硅 烷化试剂来修饰TS-1表面, 接触角测试表明催化剂的 疏水性得到了显著提高(图5a). 在丙烯环氧化反应中, 具有强疏水性的硅烷化TS-1 展现出优越的丙烯环氧化 性能: 过氧化氢转化率达到 $99.72 \%$, 环氧丙烷选择性 达到 $91.33 \%$, 并且环氧丙烷的浓度达到 $0.85 \mathrm{~mol} / \mathrm{L}$. 采 用硅烷化试剂改性有利于产物环氧丙烷的快速脱附,
从而降低环氧丙烷副产物在孔道内的累积, 有效提高 丙烯环氧化催化剂的稳定性.

与硅烷化处理不同的是, 氟化铵处理不需要引入 新的基团来取代表面硅羟基, 钛硅分子篎疏水性的提 高是通过硅羟基之间自缩合效应所实现. 这种现象主 要是通过相邻硅羟基之间的相互缩合形成硅氧桥键导 致的 ${ }^{[78]}$ (表面相邻硅羟基之间的化学反应: $(\mathrm{SiO})_{3} \mathrm{Si}-$ $\left.\mathrm{OH}+(\mathrm{SiO})_{3} \mathrm{Si}-\mathrm{OH}=(\mathrm{SiO})_{3} \mathrm{Si}-\mathrm{O}-\mathrm{Si}(\mathrm{SiO})_{3}+\mathrm{H}_{2} \mathrm{O}\right)$. 由于不 需要在表面引入新的基团, 硅羟基之间的自缩合效应 可以有效降低烯烃反应物吸附和扩散的空间位阻，从 而在改善反应物脱附的同时, 提高钛位点的可接触性. 因此, 氟化铵处理不仅能通过改善钛硅分子篮疏水性 来提高环氧化产物的选择性, 还能通过暴露更多的钛 位点来提高烯烃环氧化的活性. $\mathrm{Na}$ 等 ${ }^{[78]}$ 采用氟化铵处 理具有 $2 \mathrm{~nm}$ 厚度的片层状TS- 1 钛硅分子篮来降低片层 表面大量暴露的硅羟基的数目. 魔角旋转核磁共振 $\left({ }^{19} \mathrm{~F}\right.$ MAS NMR)结果表明, 经过氟化铵处理后的片层分子 篮没有检测到氟原子的信号, 因此硅羟基数目的减少 可以归结为相邻硅羟基之间发生缩合形成硅氧桥(如 $\left.(\mathrm{SiO})_{3} \mathrm{Si}-\mathrm{O}-\mathrm{Si}(\mathrm{SiO})_{3}\right)$. 相比于未经过处理的片层状TS1 , 经过氟化铵处理的片层状TS-1在环辛烯环氧化反 应中展现出更高的烯烃转化率和环氧化产物选择性, 这主要是归结于疏水性的提高促进了环氧化产物的脱 附, 进而减少了环氧化产物的开环副反应. 氟化铵处理 可以促进相邻硅着基 $(\mathrm{Si}-\mathrm{OH})$ 缩合生成 $\mathrm{Si}-\mathrm{O}-\mathrm{Si}$ 键，从 而有效提高钛硅分子篎的疏水性, 进而促进环氧化产 物的脱附, 减少开环等副反应的发生, 提高烯烃环氧 化选择性; 同时 $\mathrm{Si}-\mathrm{OH}$ 的减少可以有效降低孔道中的 位阻, 促进烯烃反应物的扩散, 改善钛位点和烯烃反 应物的接触, 从而提高烯烃环氧化的活性.

此外，对于工业合成过程中挤条成型催化剂的疏 水性调控具有更加实际的工业意义. Song等 ${ }^{[79]}$ 采用 $\mathrm{TPAOH}$ 水热处理TS- $1 / \mathrm{SiO}_{2}$ 挤出物, 减少了 $\mathrm{TPAOH}$ 处 理过程中对骨架钛物种的直接破坏，不仅抑制了 $\mathrm{TiO}_{2}$ 的生成, 而且有效减少了表面硅羟基与缺陷位数量, 从而有效提高了 $\mathrm{TS}-1 / \mathrm{SiO}_{2}$ 挤出物的疏水性. 在丙烯液 相环氧化反应 $648 \mathrm{~h}$ 后, $\mathrm{TS}-1 / \mathrm{SiO}_{2}$ 成型催化剂的环氧丙 烷选择性依然维持在 $99 \%$.

\section{2 调控钛物种及钛物种周边酸碱性}

降低钛物种周围环境的酸性可以有效抑制过氧化 
氢的分解以及环氧化产物的进一步开环，从而有效提 高烯烃环氧化的活性和选择性．钛硅分子笁的氮化可 以有效降低分子篮的酸性，这种方法主要是通过将氮 原子插入到钛硅分子篮的骨架中实现. 目前，最常用 的氮化方法主要是将钛硅分子篮在氨气气氛的条件下 通过高温焙烧所实现 ${ }^{[80]}$. $\mathrm{Li}^{\text {等 }}{ }^{[81,82]}$ 发现, 在氨气气氛下 低于 $1073 \mathrm{~K}$ 时，分子篎表面的 $\mathrm{T}-\mathrm{OH}(\mathrm{T}=\mathrm{Si}$ 或者 $\mathrm{Ti})$ 基团 可以被 $\mathrm{T}-\mathrm{NH}_{2}$ 所取代，而高于 $1073 \mathrm{~K}$ 时分子篮骨架中 的T-O-T可以被T-NH-T所取代(图5b). 被含N基团所修 饰的钛硅分子篮不仅具有更低分子篮的酸性，抑制过 氧化氢的分解以及环氧化产物的开环，还具有更高的 Ti-OOH中间体稳定性(图5c)，最终导致1-已烯的环氧 化转化率与选择性的提高. 然而, 氨气气氛下的高温 焙烧通常会不可避免地造成TS-1骨架结构的破坏，进 而降低TS-1催化剂的结晶度.

除表面氮化外, 本研究团队 ${ }^{[83}$ 通过构建TS-1@S-1 核壳结构(图5d)并利用特定设计的树突状 $\mathrm{Si}$ 壳来屏蔽
TS-1核上的缺陷Ti-OH，降低催化剂上的弱酸性位点 (图5e)，从而在保证Ti活性位点不被过多屏蔽时抑制 了环氧丙烷的开环副反应，进而有效提高了丙烯环氧 化的选择性. 除了采用氮化和构筑核壳结构的方法来 降低TS-1钛硅分子篮的酸性外，直接在分子篮中添加 碱性成分也可以有效地减少TS-1的酸性位. 特定的离 子 (如 $\mathrm{Na}^{+} 、 \mathrm{~K}^{+} 、 \mathrm{NH}_{4}{ }^{+}$和 $\mathrm{SO}_{4}{ }^{2-}$ ) 可以直接作为酸性抑制 剂来使用，其可以通过限制环氧化产物的开环反应来 显著提高烯烃环氧化的选择性. $\mathrm{Su}$ 等 ${ }^{[84]}$ 采用 $\mathrm{Na}^{+}$和 $\mathrm{K}^{+}$浸渍可以有效降低TS-1 钛硅分子篮的酸性, 从而提 高丙烯环氧化的反应选择性. 此外, 他们发现, 采用硫 酸盐浸渍法会明显抑制非晶态 $\mathrm{TiO}_{x}$ 物种分解过氧化氢 的副反应, 从而有效提高丙烯环氧化的活性. 紫外共振 拉曼光谱(UV resonance raman spectrum)表明, $\mathrm{TiO}_{x}$ 物 种中的 Ti-O-Ti键强度在硫酸盐浸渍之后明显减弱(图 $5 f)$, 并且过氧化氢的分解速率明显降低. 这是由于硫 酸盐和 $\mathrm{TiO}_{x}$ 物种之间通过硫酸化可以促进稳定的 $\mathrm{Ti}-$ (a)

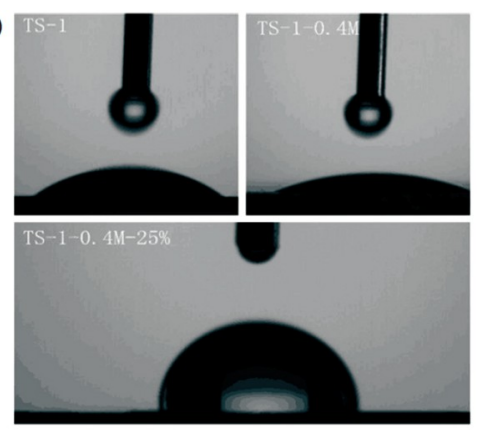

(d)

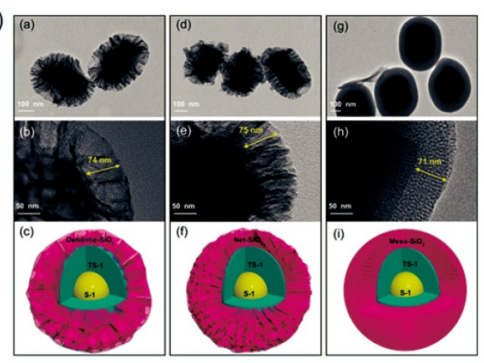

(b)

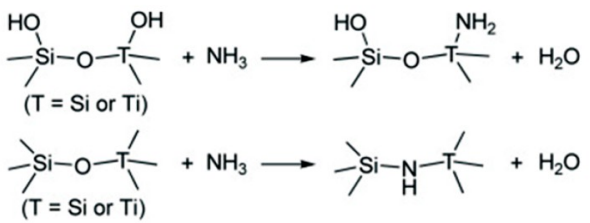

(c)

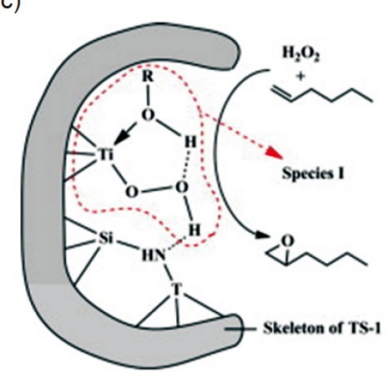

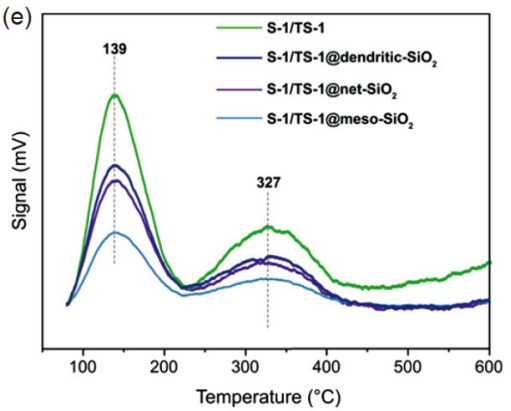

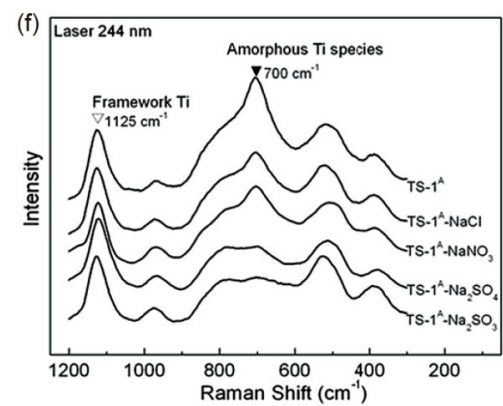

图 5 (a) TS-1在硅烷化之前和之后的接触角照片 ${ }^{[77]}$; (b) 低温和高温下的TS-1氮化过程 ${ }^{[81]}$; (c) 1-已烯和过氧化氢在N-TS-1上 的环氧化模型 ${ }^{[82]}$; (d) 不同三层结构的钛硅分子缆透射电子显微镜(TEM) 图和模型 ${ }^{[83]}$; (e) 不同三层结构的钋硅分子篮 $\mathrm{NH}_{3}-$ TPD谱图 ${ }^{[83]}$; (f) 不同溶液处理TS-1后的紫外共振拉曼谱图 ${ }^{[84]}$ (网络版彩图)

Figure 5 (a) The images of contact angle for TS-1 before and after silanization [77]; (b) the nitridation of TS-1 at higher and lower temperature [81]; (c) the model of epoxidation between the 1-hexene and $\mathrm{H}_{2} \mathrm{O}_{2}$ on the N-TS-1 [82]; (d) transmission electron microscopy (TEM) images and schematic of three-layer core-shell titanium silicalite [83]; (e) the $\mathrm{NH}_{3}$-TPD spectra of three-layer core-shell titanium silicalite [83]; (f) the UV resonance Raman spectra after impregnation with $\mathrm{NaCl}, \mathrm{NaNO}_{3}, \mathrm{Na}_{2} \mathrm{SO}_{4}$, and $\mathrm{Na}_{2} \mathrm{SO}_{3}$ [84] (color online). 
$\mathrm{O}-\mathrm{S}$ 络合物的形成, 从而可以进一步减少 $\mathrm{TiO}_{x}$ 物种中 的酸性位. Chen等 ${ }^{[85]}$ 发现, 相比于 $\mathrm{Na}^{+}$而言, 在TS-1铁 硅分子篎中添加 $\mathrm{NH}_{4}^{+}$更能够有效减少 TS- 1 的酸性，抑 制环氧丙烷的开环分解副反应，从而有效提高丙烯环 氧化活性与选择性.

针对上述酸碱性调控策略, 需要准确获得材料表 面的酸碱性信息。目前, 常用氨气程序升温吸脱附 $\left(\mathrm{NH}_{3}-\mathrm{TPD}\right)$ 和吡啶红外(Py-IR)来测定分子笁的总酸量 (强酸弱酸)和总体的B酸与 $\mathrm{L}$ 酸. 近来, 三甲基吡啶红外 由于采用的三甲基吡啶探针分子动力学直径 $(0.74 \mathrm{~nm})$ 大于吡啶分子 $(0.57 \mathrm{~nm})$, 因此其被用来表征催化剂的 外表面以及孔口的酸性位 ${ }^{[86-88]}$, 区分了分子篮型催化 剂中的微孔酸性位和外表面酸性位.

\section{4 纳米颗粒中的传质扩散}

自 1983年Taramasso 等 ${ }^{[89,90]}$ 首次通过水热法成功 合成TS-1微孔钛硅分子篮之后，一系列具有各种晶体 结构的微孔钛硅分子篮(如TS-2、Ti- $\beta$ 、Ti-ZSM-48和 Ti-FER等)被相继合成 ${ }^{[91 ~ 96]}$. 这些铁硅分子篮具有结晶 度高、四配位钛活性中心高度分散以及微孔孔道结构 可调等特点. 其中, TS-1 以其优越的反应性能得到了广 泛的应用 ${ }^{[52,97,98]}$. 但是，受制于 TS- 1 铁硅分子節 $0.54 \mathrm{~nm} \times 0.56 \mathrm{~nm}$ 的微孔孔径 ${ }^{[99 \sim 101]}(<2 \mathrm{~nm})$, 烯烃相关 反应物和产物(尤其是大分子烯烃)的传质扩散受到极 大限制。这一方面会减少烯烃反应物与四配位钛活性 中心的接触几率, 降低催化剂的反应活性; 另一方面, 狭窄的微孔孔道会极大地限制反应产物的扩散，导致 产物发生深度反应并增加TS-1催化剂的结焦倾向，导 致催化反应快速失活. 传质上的弊端极大地限制了 TS-1在烯烃环氧化中的应用. 因此, 针对TS- 1 纳米颗 粒中的传质扩散问题，提高钛活性中心位点可接触性 包括改善钛物种分布、构造复合结构的多级孔道和调 控TS-1晶粒尺寸.

\section{1 改善钛物种的分布}

改善钛物种的空间分布可以选择性地控制烯烃环 氧化反应的空间位置, 缩短环氧化产物的扩散路径并 抑制其发生开环低聚等副反应，强化烯烃环氧化反应 的选择性与稳定性. 由于核壳结构中核和壳的钛含量 明显不同，因此构造具有核壳结构的钛硅分子篮会显
著改变钛物种的分布。近来，通常采用高分辨TEM (HETEM)和Mapping耦合来定性给出催化剂总体的钛 分布 ${ }^{[102,103]}$, 而催化剂切片技术 ${ }^{[104]}$ 和电子探针显微分 析技术(EPMA)耦合则可以给出定量的钛分布 ${ }^{[105]}$.

本研究团队 ${ }^{[69]}$ 构造了具有Silicalite-1核和TS-1壳 的Au/S-1@TS-1复合结构(图6a), 该结构促使烯烃在 TS-1壳中发生环氧化反应，抑制环氧化产物在相对惰 性的Silicalite-1核中发生开环等副反应. 这种特定的核 壳结构不仅显著缩短了环氧化产物的空间扩散路径, 抑制了其在惰性的核上发生开环等副反应，从而有效 地改善了丙烯环氧化的选择性和稳定性. 除了核壳结 构外, 前述的逆向聚合法可以改变钛物种的分布，合 成的分子篮外表面具有更低的钛密度 ${ }^{[57]}$. 这种相对惰 性的外表面有效地抑制了环氧丙烷在扩散过程中的开 环, 因此具有更高的丙烯环氧化选择性.

\section{2 构造具有复合结构的多级孔道}

国际纯粹化学与应用化学会(IUPAC)根据多孔材 料的孔径大小, 将孔径分为微孔 $(<2 \mathrm{~nm})$ 、介孔 $(2 \sim 50 \mathrm{~nm})$ 以及大孔 $(>50 \mathrm{~nm})^{[106,107]}$. 既含有微孔孔道又 含有介孔孔道或者大孔孔道的复合结构被称为多级孔 道结构 ${ }^{[108]}$. 含有这种多级结构的分子篮可以有效改善 反应物和产物扩散时的空间位阻, 提高分子的扩散速 率，促使更多的钛活性中心与反应物接触，进而提高烯 烃环氧化反应速率. 目前, 多级孔道针硅分子篮的合成 方法主要包括硬模板法、软模版法以及无介孔模板法.

\subsection{1 硬模板法}

硬模板法是指将具有相对稳定结构的固体物质与 分子筷溶胶通过物理混合的方法形成组成均一的混合 晶化前驱体, 然后随着晶化的不断进行, 分子篮晶体在 固体物质周围不断生长, 直至包覆, 然后通过高温焙烧 除去晶体内的固体物质，从而形成多级孔道的方 法 $^{[109]}$. 硬模板法中通常可以采用碳模板、高分子聚合 物、无机物、有机物和生物质等物质来作为硬模板 剂 $[110]$.

碳模板以其易得易用且容易高温去除的优点，在 钛硅分子篮制备中应用广泛 ${ }^{[111,112]}$. Xin等 ${ }^{[113]}$ 采用碳模 板做介孔模板剂一步合成了具有贯穿整个晶体结构的 无序网状式介孔TS-1钛硅分子篮. 他们采用变温 ${ }^{129} \mathrm{Xe}$ NMR表征分子篮介孔孔道, 并且将低温 $173 \mathrm{~K}$ 下于 
(a)

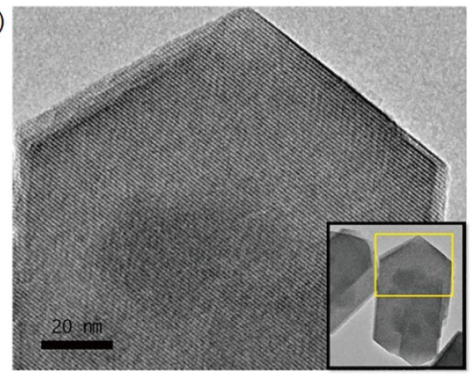

(d)

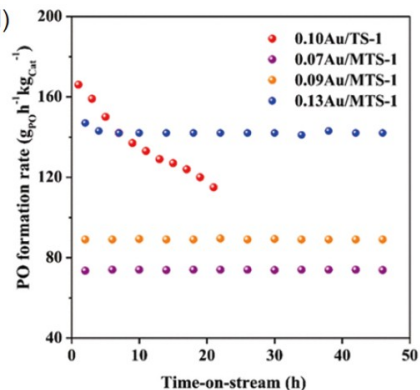

(b)

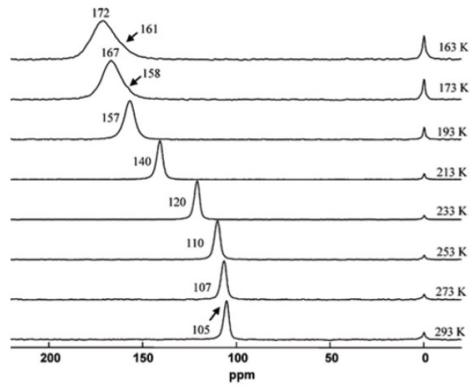

(e)

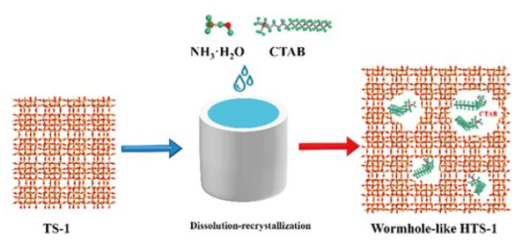

(c)

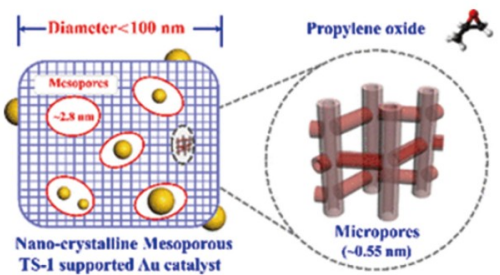

(f)

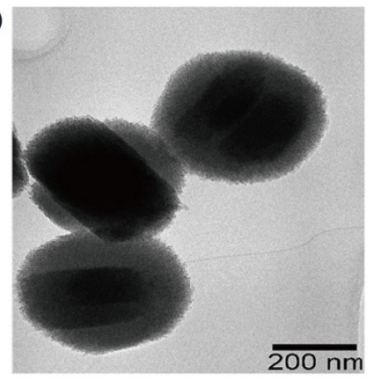

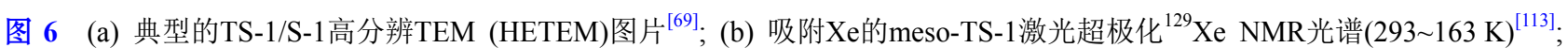
(c) 负载金颗粒的纳米介孔TS-1 示意图 ${ }^{[76]}$; (d) Au/TS-1 和Au/MTS-1 催化剂在不同反应时间下的环氧丙烷生成速率图 ${ }^{[76]}$; (e) 虫 洞型介孔TS-1的合成示意图 ${ }^{[68]}$; (f) 经过TPOAC修饰的TS-1铁硅分子笁在晶化三天后的TEM图 ${ }^{[24]}$ (网络版彩图)

Figure 6 (a) The typical high-resolution TEM (HRTEM) image of TS-1/S-1 [69]; (b) the laser-hyperpolarized ${ }^{129}$ Xe NMR spectra of Xe adsorbed in meso-TS-1 model (293-163 K) [113]; (c) the schematic of nano-crystalline mesoporous TS-1 supported Au catalyst [76]; (d) the PO formation rate of Au/TS-1 and Au/MTS-1 catalysts at different time-on-stream [76]; (e) the schematic of synthetic process for the wormhole-like TS-1 [68]; (f) the typical TEM image of TS-1 decorated by TPOAC after crystallization for three days [126] (color online).

$158 \mathrm{ppm}$ 处的峰归属于Xe原子与介孔孔道相互作用的 结果(图6b). 这种变温 ${ }^{129} \mathrm{Xe} N \mathrm{NMR}$ 为分子篮多级孔道的 表征提供了新的证明思路. 此外, 紫外-可见光光谱与 紫外-拉曼光谱表明，具有贯穿式介孔的TS-1与传统 TS-1具有相似的钛配位环境，均含有孤立的四配位骨 架钛, 并且不含有可促使过氧化氢分解的二氧化钛. 反应结果表明，具有贯穿式介孔的钛硅分子篮相比于 传统TS-1反应活性高出将近一倍, TOF值从 $15 \mathrm{~h}^{-1}$ 提高 到 $25 \mathrm{~h}^{-1}$. TS-1优越的催化活性归结于贯穿式的介孔孔 道, 其可以促进更多的反应物与活性中心钛接触, 从而 提高反应性能. Schmidt等 ${ }^{[114]}$ 采用 $18 \mathrm{~nm}$ 的碳黑做硬模 板来精准控制介孔孔径并成功合成出具有约 $20 \mathrm{~nm}$ 孔 径的介孔, 该介孔TS-1催化剂在环已烯环氧化反应中 展现出比传统TS-1高近10倍的反应活性. 与碳模板相 比，高分子聚合物(如聚合物纤维和聚合物膜)和无机 物 (如胶体晶体)在大孔分子篮的合成中更加高 效 ${ }^{[115 \sim 118]}$.

除了直接使用碳模板、高分子聚合物和无机物以 外，通过廉价易得的碳水化合物(如糖类)的原位直接
碳化得到碳模板的方法引起了人们的广泛关注. Wang 等 ${ }^{[111]}$ 采用廉价的 TPABr和蔗糖分别作为微孔和介孔 模板剂来合成具有多级孔道的TS-1. 结果表明, 该分子 篮不仅具有有序的微孔孔道和晶内互联的介孔孔道, 还同时具有一部分大孔孔道. 这些介孔和大孔孔道的 形貌与限域在分子篮内部由蔗糖碳化生成的碳的形貌 相似, 表明介孔和大孔来源于蔗糖的碳化. TS- 1 的多级 孔道可以显著缩短微孔孔道内部分子的运动路径, 同 时降低分子扩散的空间位阻，进而有效改善反应物分 子的晶内扩散, 从而提高催化反应活性. 因此, 在扩散 控制时，提高催化活性位的可接触性比提高本征活性 更加重要.

不同于以上方法，生物质模板不仅可以高效制备 多级孔道，而且可以同时通过生物质本身的大量官能 团来修饰催化剂的表面 ${ }^{[19]}$, 这些官能团拥有广阔的应 用前景, 包括能源、催化、传感器和生物医药等方面. Yuan等 ${ }^{[120]}$ 采用聚二烯丙基二甲基氯化铵(PDDA)改性 的硅藻土为模板, 将含有TPAOH (模板剂)、TEOS (硅 源)、TBOT (钛源)的溶胶直接与硅藻土混合并原位晶 
化, 从而得到TS-1/硅藻土复合物催化剂. 新合成的硅 藻土催化剂具有丰富的多级孔道, 比表面积高达 $521.3 \mathrm{~m}^{2} / \mathrm{g}$, 并且微孔孔容达到 $0.254 \mathrm{~cm}^{3} / \mathrm{g}$. Liu等 ${ }^{[30]}$ 通 过硅藻土的预处理来改善TS-1/硅藻土复合物催化剂 的反应性能. 通过 $\mathrm{H}_{2} \mathrm{SO}_{4} / \mathrm{HF}$ 混合酸溶液的预处理来脱 除硅藻土表面的金属杂质(如 $\mathrm{Al} 、 \mathrm{Fe} 、 \mathrm{Mg}$ )并清除孔 道内的堵塞物. 此外, 经过酸处理的硅藻土表面酸强度 降低, 但酸位点数量增多. 该TS-1/硅藻土复合物催化 剂有利于催化剂的后续分离以及工业成本的降低.

\subsection{2 软模版法}

和硬模板法不同的是, 软模版法采用结构相对柔 韧可溶的分子 ${ }^{[121]}$, 该软模版分子与分子篮溶胶中的分 子存在各种相互作用, 可以是作用力较强的化学键作 用力也可以是作用力相对较弱的电荷相互作用力和氢 键作用力. 但是, 用于合成微孔孔道的有机模板剂和用 于合成介孔孔道的软模板剂通常存在竞争关系, 而不 是协同关系, 并且二者在高温、高压和强碱的晶化条 件下存在晶化动力学的差异. 这些特点容易导致微孔 相和介孔相分离, 不利于贯通式多级孔道钛硅分子篮 的形成 ${ }^{[109]}$. 为避免这种情况的发生, 目前在钛硅分子 管合成时常用的软模板剂包括可溶聚合物 ${ }^{[98,122]}$ 、表 面活性剂 ${ }^{[123]}$ 以及有机硅烷试剂 ${ }^{[124]}$.

采用可溶聚合物不仅可以合成具有多级孔道的 $\mathrm{TS}-1$, 同时可以有效减小钛硅分子篎的粒径. Du等 ${ }^{[122]}$ 采用环保且廉价的聚合物(聚醋酸乙烯酯, PVA), 通过 三步晶化法成功合成出由 $30 \mathrm{~nm}$ 左右的超小粒径TS-1 聚集形成的具有多级孔道的 TS-1 纳米团簇. 聚合物 PVA通过堆叠超小 TS-1 晶体来实现晶间介孔的构筑, 从而在有效减小分子扩散路径的同时也显著减小分子 扩散的空间位阻.

除采用聚合物为软模板剂外, 具有特定亲水亲油 基团的表面活性剂由于在溶液中特殊的聚集行为引起 了广泛关注 ${ }^{[123,125,126]}$. 本研究团队 ${ }^{[76]}$ 以廉价非离子表 面活性剂P123为介孔模板剂, 采用干胶法成功合成出 具有均一介孔 $(\sim 2.8 \mathrm{~nm})$ 的纳米晶钛硅分子篮 $(<100 \mathrm{~nm})$, 如图 $6 c$ 所示. 该载体优越的疏水性和微介 复合多级孔道有效改善了反应物和产物的空间扩散性 能, 同时提高了丙烯环氧化反应的稳定性和选择性, 如 图6d所示. Du等 ${ }^{[98]}$ 采用聚二烯丙基二甲基氯化铵 (PDADMAC) 作为介孔模板剂通过水热合成法成功合
成出具有丰富介孔 $(5 \sim 40 \mathrm{~nm})$ 的多级孔道TS-1. 通过优 化合成溶液中的溶液配比 $\left(\mathrm{SiO}_{2}: 0.033 \mathrm{TiO}_{2}: 0.30\right.$ TPAOH: $\left.124 \mathrm{H}_{2} \mathrm{O}: 0.085 \mathrm{PDADMAC}\right)$ 可以合成具有更加 均一介孔( 10 nm) 的TS-1催化剂.

采用有机硅烷试剂作介孔软模板剂可以有效避免 微孔相和介孔相的分离, 这是由于有机硅烷中的硅原 子可以参与到分子篮骨架的搭建当中, 从而有效连接 微孔孔道和介孔孔道, 起到“穿针引线”的作用. 需要 注意的是, 不同于通过硅烷化试剂来调变TS-1疏水性 的方式, 这里采用的软模板法是在分子篮水热合成时 原位添加有机硅烷试剂. Cheneviere等 ${ }^{[124]}$ 使用有机硅 烷偶联剂二甲基十八烷基 [3-(三甲氧基硅基)丙基]氯 化铵(TPOAC) 作为介孔模板剂, 成功合成了具有微介 复合的多级孔道TS-1 (图6f), 并且该催化剂展现出相 对于普通TS-1更优越的环已烯环氧化活性. 有机硅烷 在作为介孔模板剂的同时, 可以有效修饰TS-1催化剂 表面, 逐步取代表面硅羟基进而提高催化剂疏水性. Serrano 等 ${ }^{[70]}$ 发现，有机硅烷试剂苯基胺丙基三甲氧基 硅烷(PHAPTMS)在分子篎成核初期通过 $\mathrm{Si}-\mathrm{O}-\mathrm{Si}$ 键之 间的缩合使有机硅烷分子插入到分子篮中，从而抑制 与其他硅源之间的进一步缩合反应. 该方法有效抑制 了 TS-1铁硅分子篮的熟化生长, 将TS-1钛硅分子篮的 尺寸成功控制在纳米尺度. 相比于普通 TS-1, 该多级 孔道纳米TS-1可以显著提高1-辛烯和环已烯环氧化的 TOF值, 并且仍然能保持 $100 \%$ 的高选择性. 因此, 在采 用有机硅烷试剂做TS-1介孔软模板剂时, 不仅可以构 造微介复合的多级孔道, 同时可以有效改善表面疏水 性并抑制TS-1钛硅分子篮的熟化生长.

\subsection{3 无介孔模板法}

相较于硬模板法和软模版法, 无介孔模板剂法最 大的特点是只需要使用一种特定的微孔模板剂而不再 需要同时使用微孔和介孔两种模板剂来构筑TS-1多级 孔道, 从而有效节约资源, 减少环境污染 ${ }^{[119]}$. 典型的无 介孔模板法主要包括双功能模板法、晶种辅助法以及 刻蚀法.

双功能模板法指的是微孔模板剂不单单可以用来 合成某一种微孔孔道, 其可以同时用来构筑微孔、介 孔甚至大孔孔道. 特殊设计的表面活性剂可以同时作 为合成微孔和介孔的模板剂, 而不再需要添加额外的 介孔模板剂. $\mathrm{Na}$ 等 ${ }^{[78]}$ 合成并采用双季铵盐表面活性剂 
$\left(\mathrm{C}_{16} \mathrm{H}_{33} \mathrm{~N}+\left(\mathrm{CH}_{3}\right)_{2} \mathrm{C}_{6} \mathrm{H}_{12} \mathrm{~N}+\left(\mathrm{CH}_{3}\right)_{2} \mathrm{C}_{6} \mathrm{H}_{13}\right)$ 作为结构导向 剂成功合成出纳米片层堆叠的 TS-1 钛硅分子篮(图7a). 受到双季铵盐模板剂的导向作用, 纳米片层钣硅分子 篮沿 $a c$ 平面向外扩展生长, $b$ 方向高度仅为 $2 \mathrm{~nm}$ 的单 胞厚度(图 $7 \mathrm{~b}, \mathrm{c}$ ). 片层TS-1自身具有约 $0.55 \mathrm{~nm}$ 微孔孔 道, 堆叠的片层结构则形成晶间介孔孔道. 因此片层结 构能够暴露大量的环氧化活性中心(四配位骨架钛位 点)并有效减少反应物扩散的空间位阻, 进而有效催化 大分子烯烃环氧化．这种新颖的合成思路提供了一种 从源头上高效合成微介复合多级孔道TS-1钛硅分子篮 的新方法.

Bai 等 ${ }^{[127]}$ 通过纯硅晶种辅助的方法, 成功利用中 间结晶策略合成出纳米多级孔TS-1 (图7d). 这种晶种 辅助的中间结晶策略(在分子篮完全晶化之前取出样 品)通过调控晶化时间来控制晶种之间的融合生长来 同时实现晶间介孔和晶内介孔的构筑(图7e, f), 从而有 效改善钛位点的可接触性. 其中, 晶内介孔的出现归结 于溶解重结晶过程的晶内碱腐蚀，而晶间介孔则归结 于纳米 TS-1 晶体之间融合生长过程中的相互堆叠. 中
间结晶策略有效抑制了晶种之间的过度融合，从而保 留大量的晶间介孔。这种晶种辅助的中间结晶策略, 不仅有效节约了时间成本，避免了非骨架铁的生成， 而且同时保留了 TS-1晶体中的晶内介孔和晶间介孔, 改善了反应物和产物的扩散阻力.

Chen等 ${ }^{[128]}$ 采用准固态化学结晶法来制备同时具 有微孔、介孔和大孔的等级孔钛硅分子篮(图8a, b). 该等级孔钛硅分子篮首先需要通过直接干燥钛硅混合 物合成非晶相的多级大孔-介孔钛硅前驱体. 随后将该 前驱体和模板剂、钛源和硅源混合晶化, 即可在孔壁 上进一步生长微孔钛硅分子篮，从而形成具有微孔、 介孔和大孔的等级孔钛硅分子管. TS-1催化剂显示出 均一的大孔结构和高度互连的介孔网络, 并且该网络 由粒径均匀的钛硅分子篮纳米晶体构成. 与常规TS-1 纳米晶体相比, 这种新颖的等级孔结构减小了烯烃反 应物分子扩散的空间位阻，改善了铁位点的可接触性， 使催化剂表现出优异的催化性能, 尤其是对于大分子 烯烃的环氧化反应. 此外, 这种准固态化学结晶法可 以用来合成一系列具有微孔-介孔-大孔的多级孔道分 (a)

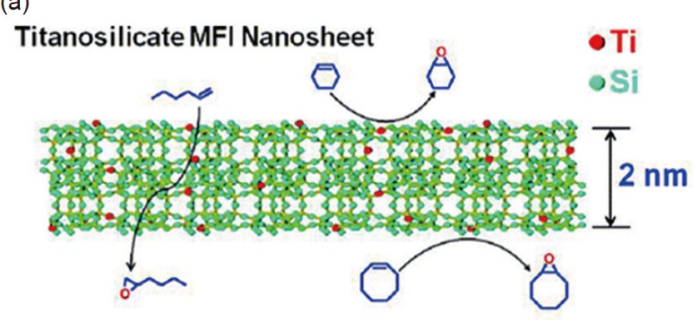

(d)

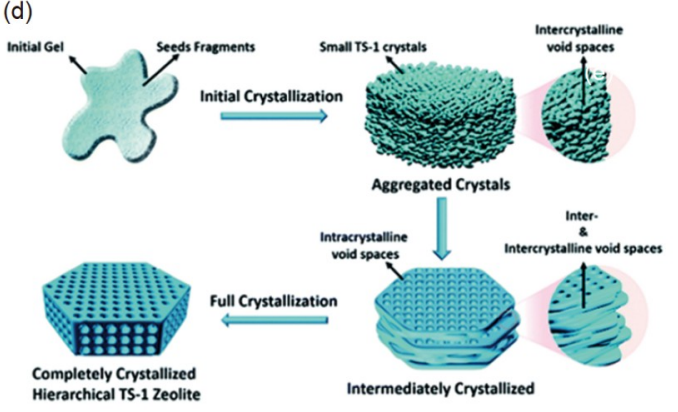

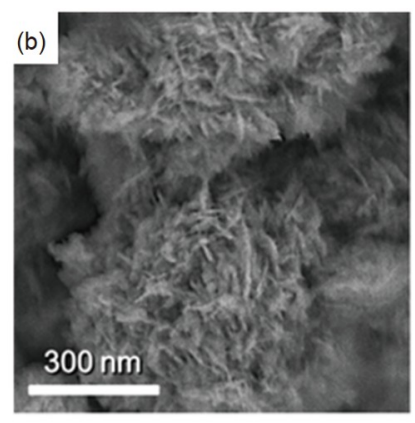

(e)

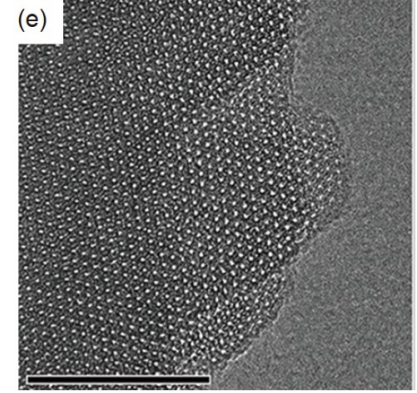

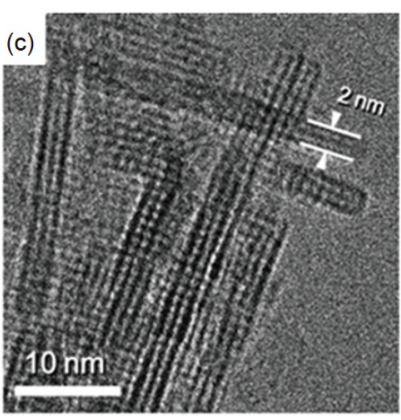

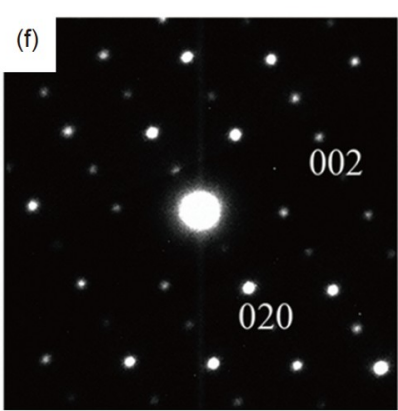

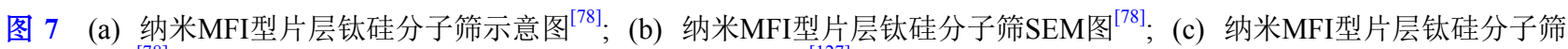
HRTEM 图 $^{[78]}$; (d) 中间结晶和完全结晶的TS-1催化剂合成过程 ${ }^{[127]}$; (e) 晶化3 h的纳米介孔TS-1催化剂HRTEM图, 标尺为 $20 \mathrm{~nm}^{[127]}$; (f) 晶化 $3 \mathrm{~h}$ 的纳米介孔TS-1催化剂选区电子衍射图 ${ }^{[127]}$ (网络版彩图)

Figure 7 (a) The schematic of titanosilicate MFI nanosheet [78]; (b) the typical SEM image of titanosilicate MFI nanosheet [78]; (c) the typical HRTEM image of titanosilicate MFI nanosheet [78]; (d) the synthesis of the completely crystallized and intermediately crystallized TS-1 catalysts [127]; (e) the typical HRTEM image of nano-sized hierarchical TS-1 after crystallization for $3 \mathrm{~h}$ (scale bar, $20 \mathrm{~nm}$ ) [127]; (f) the selected area electron diffraction patterns of nano-sized hierarchical TS-1 after crystallization for $3 \mathrm{~h}$ [127] (color online). 
(a)

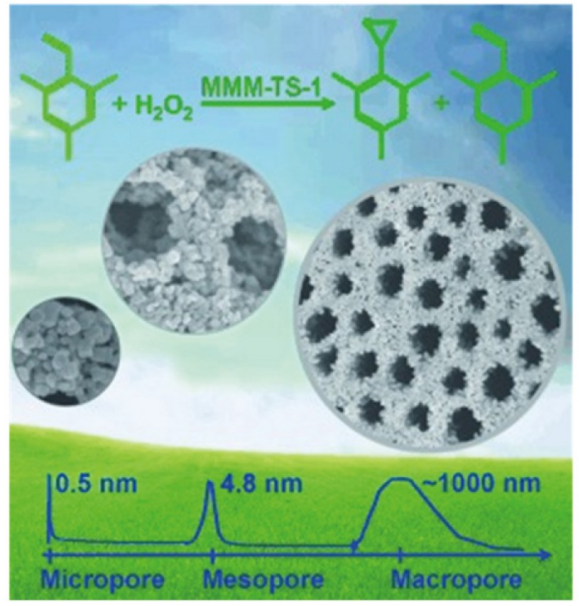

(b)

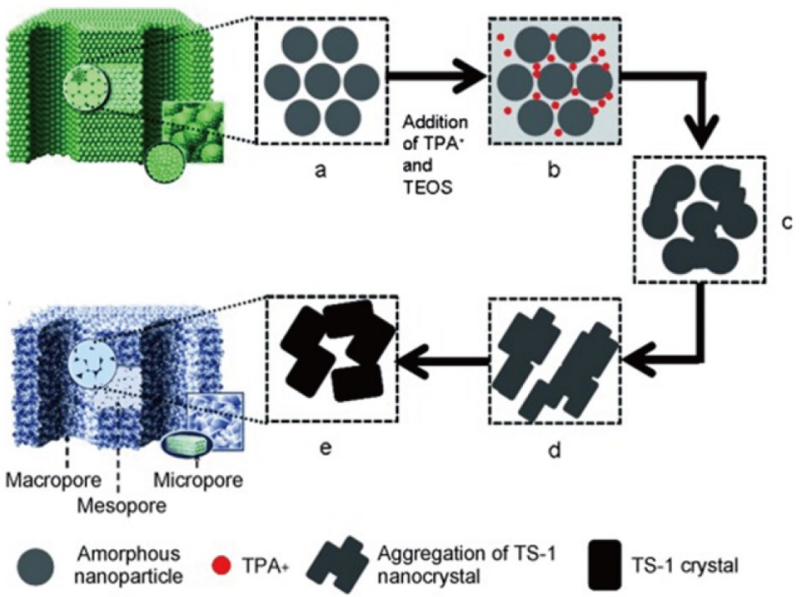

图 8 (a) 微孔-介孔-大孔TS-1结构示意图 ${ }^{[128]}$; (b) 微孔-介孔-大孔TS-1合成机理图 ${ }^{[128]}$ (网络版彩图)

Figure 8 (a) The structural schematic of micro-meso-macroporous TS-1 catalyst [128]; (b) the mechanism of synthesizing micro-meso-macroporous TS-1 catalyst [128] (color online).

子笁催化剂.

多级孔道分子篮的合成策略不断迭代更新，同时 多级孔道的表征方法也得到了相应的发展. 相比于单 纯表征介孔孔道的变温 ${ }^{129} \mathrm{Xe} \mathrm{NMR}$, 新兴的脉冲梯度 场-核磁共振(PFG-NMR)为表征TS-1 钛硅分子篮多级 孔道的互联性提供了一种可行的方法 ${ }^{[129,130]}$. 未来, 以 钛硅分子篮为催化剂的扩散动力学表征可以分别从实 验测试(如微量分析和红外光谱的耦合 ${ }^{[86]}$ )和模拟计算 (如分子动力学计算 ${ }^{[131]}$ )两个方面来深入研究分析.

无模板法中应用最广泛的方法就是刻蚀法. 该方 法操作简单、重复性好, 并且能够通过晶体刻蚀生成 大量介孔甚至大孔. 其中最常用的刻蚀试剂为碱性试 剂, 包括 $\mathrm{NaOH}$ 和TPAOH. NaOH刻蚀TS-1会选择性地 刻蚀硅原子, 因此 $\mathrm{NaOH}$ 在生成介孔孔道的同时也改 变了TS-1的硅钛比，从而影响了材料的固有属性 ${ }^{[09]}$. 不同于 $\mathrm{NaOH}$ 试剂，TPAOH刻蚀骨架中硅原子的同时， 能够在水热晶化的条件下通过重结晶的作用来重新利 用被刻蚀的硅原子和散落于分子篮周围的钛物种来构 筑孔道结构. Jiao 等 ${ }^{[132]}$ 采用 $0.5 \mathrm{M}$ TPAOH溶液原位处 理 TS-1, 成功将介孔和大孔孔容从 0.014 提高到 $0.168 \mathrm{~cm}^{3} / \mathrm{g}$ (图9a, b), 非骨架钛物种从 $14.4 \%$ 明显减少 到 $0.3 \%$ ，从而改善了环已烯扩散的空间位阻并且抑制 了过氧化氢的无效分解, 将环己烯环氧化的收率提高 将近3倍. $\mathrm{Li}$ 等 ${ }^{[133]}$ 通过TPABr和乙醇胺处理微米级的 微孔TS-1来合成多级孔道TS-1. 将该钛硅分子篮挤条 成型后，得到的催化剂具有明显的介孔和大孔结构，
从而改善反应物的扩散性能. 通过正交条件优化后的 催化剂, 具有最高的机械强度 $(200 \mathrm{~N} / \mathrm{m})$, 并且丙烯环 氧化反应后环氧丙烷的收率达到 $92.5 \%$.

由于碱性试剂的选择性刻蚀导致的TS-1材料硅钛 比改变, $\mathrm{Du}$ 等 ${ }^{[134]}$ 采用酸性试剂 $\left(\mathrm{NH}_{4} \mathrm{~F}\right.$ 和 $\mathrm{HF}$ 混合溶液 $)$ 来刻蚀TS-1中的硅原子和钛原子, 从而在保证材料中 硅钛比不变的条件下, 构建微孔/介孔/大孔共存的等 级孔TS-1 钛硅分子篮材料(图9c, d). 采用酸性试剂和 碱性试剂最大的不同在于对钛硅分子篮材料中的元素 刻蚀是否具有选择性. 采用 $\mathrm{TPAOH}$ 和 $\mathrm{NaOH}$ 等碱性试 剂会选择性刻蚀铁硅分子篮中的硅原子, 因此在刻蚀 出介孔孔道的同时也会改变载体的硅钛比. 然而采用 酸性试剂 $\left(\mathrm{NH}_{4} \mathrm{~F}\right.$ 和 $\mathrm{HF}$ 混合溶液)刻蚀硅原子的同时也 会刻蚀钛原子, 从而在生成介孔孔道的同时保证载体 硅钛比不变, 进而有效保证单一变量.

尽管刻蚀法目前取得了一系列的成功, 但是仍然 有许多问题需要关注，如由于破坏性所带来的分子篮 结晶度和相对质量的损失, 这些都是亟待进一步解决 的问题. 相比于硬模板法和软模版法, 无介孔模板法 虽然可以有效避免介孔模板剂的使用, 减少环境污染, 并且节约资源; 但是合成双功能模板剂所需的高昂的 成本和极其复杂的合成步骤仍限制了该方法的大规模 工业应用。

此外，催化剂工业合成中挤条成型的条件也会极 大地影响反应物和产物的空间扩散. Zuo等 ${ }^{[135]}$ 通过在 挤条的过程中添加SBA-15和碳化硅来改善1-丁烯环 

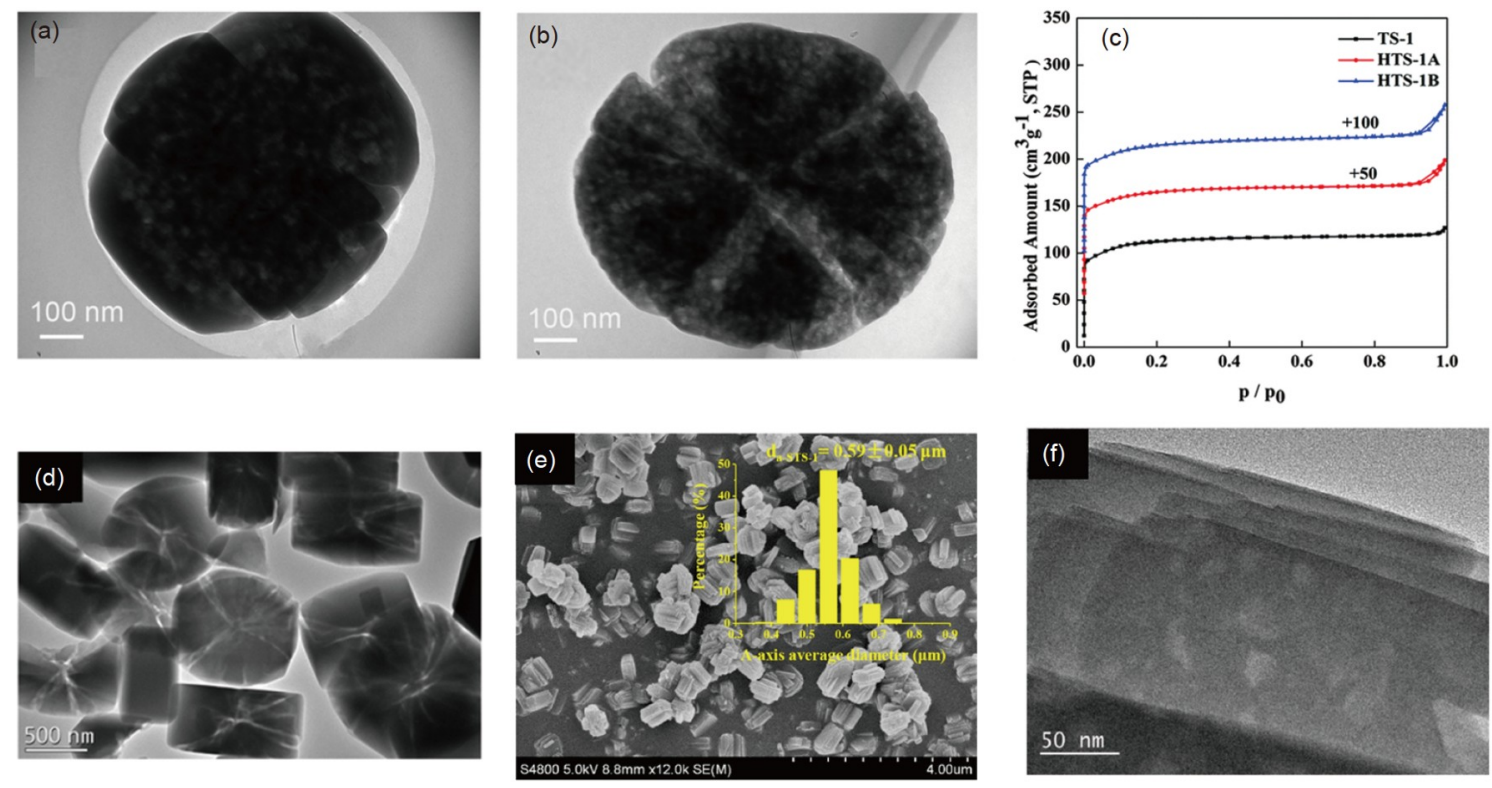

图 9 (a) $0.4 \mathrm{M}$ TPAOH处理过的TS-1催化剂TEM图像 ${ }^{[132]}$; (b) $0.5 \mathrm{M}$ TPAOH处理过的TS-1催化剂TEM图像 ${ }^{[132]}$; (c) TS-1 和具 有多级孔道的 TS-1氮气物理吸脱附曲线 ${ }^{[134]}$; (d) NaOH和 $\mathrm{NH}_{4} \mathrm{~F}-\mathrm{HF}$ 混合处理TS-1催化剂的TEM图像 ${ }^{[134]}$; (e) 蒸汽辅助法合成的 STS-1催化剂的SEM图像 ${ }^{[138]}$; (f) 蒸汽辅助法合成的STS-1催化剂的HRTEM图像 ${ }^{[138]}$ (网络版彩图)

Figure 9 (a) The typical TEM image of TS-1 treated with $0.4 \mathrm{M}$ TPAOH solution [132]; (b) the typical TEM image of TS-1 treated with $0.5 \mathrm{M}$ TPAOH solution [132]; (c) $\mathrm{N}_{2}$ adsorption/desorption isotherms of TS-1 and the hierarchical TS-1 [134]; (d) the typical TEM image of TS-1 treated with the mixture of $\mathrm{NaOH}$ and $\mathrm{NH}_{4} \mathrm{~F}-\mathrm{HF}$ [134]; (e) the representative SEM image of STS-1 synthesized by steam-assisted crystallization method [138]; (f) the representative HRTEM image of STS-1 synthesized by steam-assisted crystallization method [138] (color online).

氧化的反应性能. 1 -丁烯的转化率从 $25 \%$ 提高到 $32 \%$, 环氧化产物的选择性均接近 $100 \%$. 这是由于添加的碳 化硅可以有效提高成型催化剂的导热系数和热导率, 从而有利于热量的移出并减少副反应的发生. 此外, SBA-15的添加使成型催化剂的比表面积从 $341 \mathrm{~m}^{2} / \mathrm{g}$ 提 高到 $382 \mathrm{~m}^{2} / \mathrm{g}$, 有效改善反应物和产物在催化剂中的 扩散性能以及催化剂的容碳能力.

\section{3 调控分子篮晶体尺寸}

缩小TS-1 晶体尺寸可以有效缩短烯烃反应物和环 氧化产物在晶体内扩散的路径, 包括烯烃反应物从TS孔 孔扩散到活性 Ti位点附近、环氧化产物从活性钛 位点扩散到TS-1孔口，进而改善烯烃环氧化的活性、 选择性与稳定性. 目前, 合成小尺寸TS-1的方法主要 包括晶种法、干胶法和生长抑制剂法.

\subsection{1 晶种法}

晶种法指的是在钛硅分子篮前驱体凝胶中加入 MFI型分子篮晶种制成混合均匀的悬浊液, 然后在高
温高压下进行混合晶化 ${ }^{[136,137]}$. 接下来, 溶液中的硅源 和钛源在模板剂的作用下沿着晶核生长，而加入的大 量晶种会在分子篮晶化前期作为晶核供硅源和钛源生 长, 从而极大程度地将更多的硅源和钛源分散, 促使分 子篮晶体向小尺寸方向演化. 本研究团队 ${ }^{[138]}$ 以S-1作 为晶种, 采用廉价的模板剂(正丁胺)和硅源(硅溶胶)合 成高效廉价的具有核壳结构的TS-1/S-1 (图9e, f). 在不 添加晶种的情况下, 传统水热法采用正丁胺(模板剂) 和硅溶胶(硅源)合成出来的TS-1通常为微米尺度, 晶 体尺寸较大, 反应性能较差. 然而, 晶种的添加促使 TS-1晶体三维尺寸缩小为 $220 \mathrm{~nm} \times 130 \mathrm{~nm} \times 80 \mathrm{~nm}$, 有 效缩短了分子的扩散路径. 此外, 该分子篮还具有更 好的疏水性和一定的介孔容碳能力, 使得该TS-1/S-1 复合分子篮在丙烯环氧化中展现出超过 $100 \mathrm{~h}$ 的稳定 性. 因此, 晶种法除了可以调控分子篮晶体尺寸外, 还 可以通过使用廉价模板剂达到和传统昂贵模板剂相近 的晶体尺寸和反应性能，从而在一定程度上降低高性 能TS-1分子篎的价格, 更有利于推进TS-1的工业化 进程. 


\subsection{2 干胶法}

干胶法相较于传统水热晶化法最大的不同在于将 分离的固体干胶和液体同时晶化. 在这种晶化过程中 干胶前驱体的移动性很差，导致在干胶成核和生长的 过程中更容易相互聚合而不是相互融合并进一步熟化 长大. 因此, 这种聚集体在保证分子篮晶体尺寸较小的 同时, 可以形成大量的晶间介孔, 从而在这种分子篮聚 集体中构筑具有微介复合的多级孔道 ${ }^{[139]}$. 此外，相对 于传统的水热合成法, 干胶法还具有以下特点: 更短的 结晶时间和更少的模板剂用量. 其中, 根据模板剂在晶 化过程中位置的不同，通常将干胶法分为气相传输法 (VPT) 和蒸汽辅助转化法 $(\mathrm{SAC})^{[140 \sim 142]}$. 在气相传输法 中，下方液相的水和模板剂在高温下汽化上升与上方 的分子篎前驱体干胶相互接触晶化，而在蒸汽辅助转 化法中，下方液相中(只含有水)的液体在高温下汽化 上升与上方的分子篎前驱体干胶和模板剂的混合物相 互接触并进一步晶化. 其中, 水分子的汽化作用不仅仅 是提供TS-1 晶化过程中成核和生长所必须的自生压 力, 而且其通过参与分子鉴前驱体水解和缩合的形式
来构建TS-1钛硅分子篮骨架结构，并在其中起到至关 重要的作用.

$\mathrm{Du}$ 等 ${ }^{[139]}$ 充分研究了 $\mathrm{SAC}$ 方法中干胶的干燥温 度、水含量以及是否研磨等因素的影响. 实验发现, 干 胶的预研磨能够促使干胶结构更加松散，这种低密度 的松散结构有利于水蒸气在干胶中的扩散, 从而加速 前驱体的水解与缩合，并促进具有微介复合多级孔道 的小尺寸TS-1纳米晶聚集体(图10A，B)的形成. 此外, 由于硅物种向干胶内部迁移导致钛物种在TS- 1 表面大 量富集，从而促使TS-1的表面钛浓度要高于分子篮内 部的钛浓度. Zhou等 ${ }^{[143]}$ 采用干胶转化法成功合成出 微介复合的TS-1分子篮，该分子篮催化剂在选择性氧 化反应中展现出更高的反应活性和稳定性, 这归结于 该分子篮优异的扩散能力和较高的水热稳定性(抑制 介孔结构的坍塌和钛含量的损失). 干胶转化法不仅 可以合成尺寸更小的 TS-1 钛硅分子篮, 而且可以利用 干胶前驱体更差的移动性来合成具有大量晶间介孔 的TS-1. 更小的晶体尺寸和更多的晶间介孔有利于缩 短分子扩散的路径并降低分子扩散的空间位阻，从而

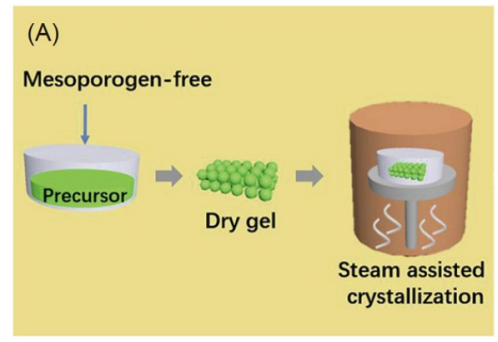

(D)

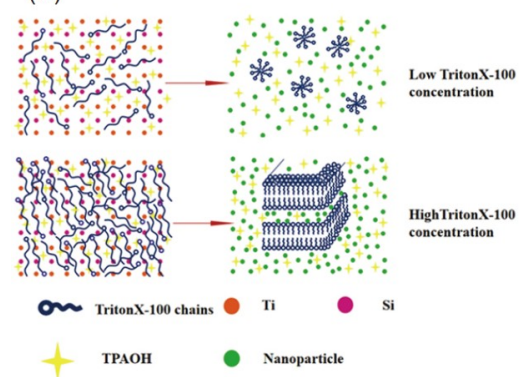

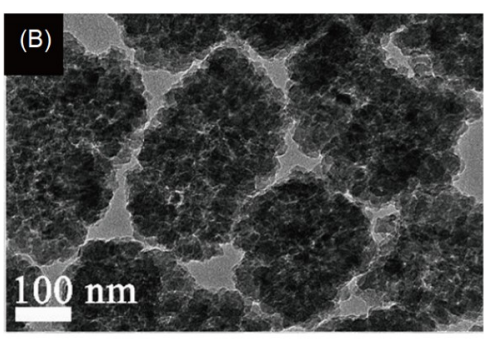

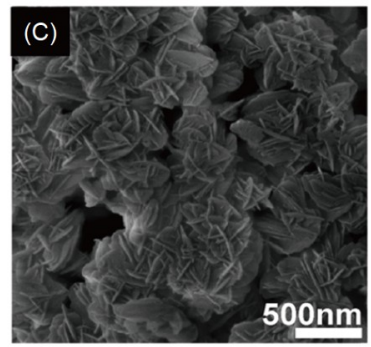

(E)
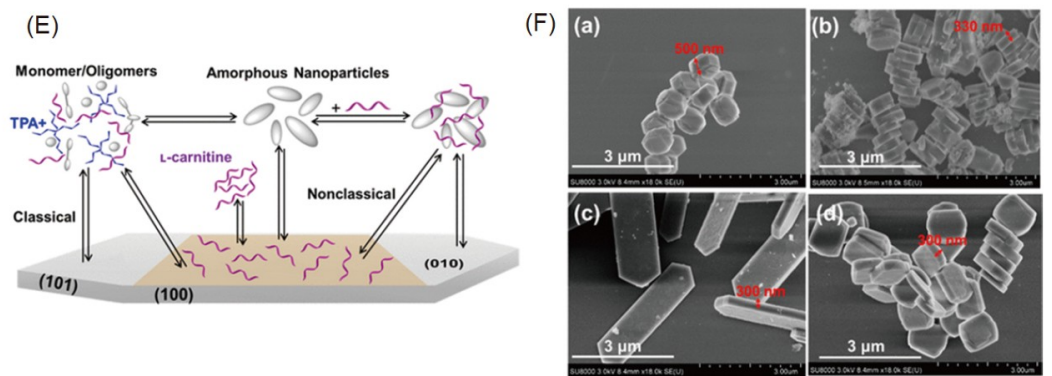

图 10 (A) 干胶转化法合成多级孔道TS-1示意图 ${ }^{[139]}$; (B) 前驱体在 $40{ }^{\circ} \mathrm{C}$ 下干燥合成的多级孔TS-1催化剂的 TEM图像 ${ }^{[139]}$; (C) 纳米片层TS-1 ( $\mathrm{Si} / \mathrm{Ti}=25)$ 催化剂的SEM图像 ${ }^{[145]}$; (D) 使用Triton X-100合成多级孔道TS-1的形成机制 ${ }^{[146]}$; (E) 赖氨酸在TS-1合 成过程中的作用机理 ${ }^{[147]}$; (F) 使用不同添加剂合成TS-1催化剂的SEM图像((a) 传统TS-1; (b) 乙醇/赖氨酸=15:0.4; (c) 乙醇/赖氨 酸 $=15: 0.6 ;(\mathrm{d})$ 乙醇/赖氨酸 $=0: 0.6)^{[147]}$ (网络版彩图)

Figure 10 (A) The schematic of synthesizing hierarchical TS-1 by dry gel conversion method [139]; (B) the typical TEM image of HTS-1-40 (precursors were dried at $40^{\circ} \mathrm{C}$ ) [139]; (C) the representative SEM image of TS-1 nanosheets $(\mathrm{Si} / \mathrm{Ti}=25)$ [145]; (D) the formation mechanism of hierarchically porous TS-1 using Triton X-100 surfactant [146]; (E) the action mechanism of lysine during the synthesis of TS-1 [147]; (F) SEM images of (a) traditional TS-1, (b) TS-1 (15Et:0.4LC), (c) TS-1 (15Et:0.6LC), and (d) TS-1 (0Et:0.6LC) (LC=lysine) [147] (color online). 
提高活性钛位点的可接触性, 改善烯烃环氧化反应 性能.

\subsection{3 添加生长抑制剂法}

在晶化凝胶前驱体中添加生长抑制剂可以有效调 控晶体尺寸. 一般地, 生长抑制剂会通过阻断已形成的 TS-1 晶体与分子篮凝胶中的硅源和钛源的缩合来抑制 TS-1的过度生长, 从而达到控制TS-1钛硅分子篮晶体 尺寸的目的. 目前, 使用比较广泛的生长抑制剂主要 是硅烷化试剂, 硅烷化试剂可以通过与硅源之间的缩 合形成Si-O-Si键插入到TS-1晶体中, 硅烷分子尾部的 烷基基团则会有效地阻碍硅源在该位点的进一步缩合 和生长, 从而起到抑制TS- 1 晶体生长的目的.

Sanz等 ${ }^{[144]}$ 通过添加硅烷化试剂苯基胺丙基三甲 氧基硅烷(PHAPTMS)将TS-1颗粒尺寸从 $1 \mu \mathrm{m}$ 左右减 小到200 300 nm, 这些200 300 nm的TS-1颗粒实际上 是由 20 40 nm的TS-1纳米晶体所构成. 这些超小的 TS-1纳米晶体是由于硅烷化试剂在晶化过程中插入到 TS-1 钛硅分子篮晶体中并抑制TS-1纳米晶的进一步生 长所形成的. 相较于传统TS-1, 该催化剂在1-辛烯和环 己烯的环氧化反应中均展现出更优越的转化率和 TOF 值. $\mathrm{Li}$ 等 ${ }^{[145]}$ 通过添加 $\left[\mathrm{C}_{6} \mathrm{H}_{13}-\mathrm{N}^{+}\left(\mathrm{CH}_{3}\right)_{2}-\mathrm{C}_{6} \mathrm{H}_{12}-\mathrm{N}^{+}\left(\mathrm{CH}_{3}\right)_{2}\right.$ $-\left(\mathrm{CH}_{2}\right)_{12}-\mathrm{O}-\left(p-\mathrm{C}_{6} \mathrm{H}_{4}\right)_{2}-\mathrm{O}-\left(\mathrm{CH}_{2}\right)_{12}-\mathrm{N}^{+}\left(\mathrm{CH}_{3}\right)_{2}-\mathrm{C}_{6} \mathrm{H}_{12}-$ $\left.\mathrm{N}^{+}\left(\mathrm{CH}_{3}\right)_{2}-\mathrm{C}_{6} \mathrm{H}_{13}\right]\left[\mathrm{OH}^{-}\right]_{4}$ 两亲分子表面活性剂来构筑十 字交叉的片层TS-1催化剂. 由于大量的烷基基团在构 筑分子篮晶体上所起到的抑制作用, 致使晶体沿 $\mathrm{b}$ 轴 方向的生长受到极大抑制, 从而构建出 $b$ 轴极小的纳 米片层TS-1晶体(图10C). 片层TS-1催化剂将环己烯和 环辛烯环氧化反应的TOF值分别从19.1和 $10.4 \mathrm{~h}^{-1}$ 提升 到 98.3 和 $75.9 \mathrm{~h}^{-1}$, 其优越的环氧化性能依赖于 $2 \mathrm{~nm}$ 厚 的薄片结构所改善的大分子反应物的扩散性能. 此外, Shen等 ${ }^{[146]}$ 通过添加表面活性剂(TritonX-100)来限制 TS-1纳米晶体与硅源和钛源的接触, 从而达到抑制TS1钛硅分子篮生长的目的. 随着TritonX-100在溶液中浓 度的提高, TritonX-100疏水性的尾部从随机排列转变 为有序朝向, 形成的胶束集团也从球形逐渐变为网状 结构，从而有效抑制了 TS-1纳米晶体之间的相互融合 长大(图10D), 进而可以将 TS-1 晶体的尺寸从 $550 \mathrm{~nm}$ 减 小到 $340 \mathrm{~nm}$. 不同于之前所采用的将TS-1纳米晶体与 硅源和钛源相隔离的策略, Song 等 ${ }^{[147]}$ 利用赖氨酸与 无形性纳米颗粒前驱体特定晶面和硅源、钛源之间的
配位作用以及在特定晶面上 ( $a c$ 平面)的覆盖来定向生 长TS-1 (图10E), 从而有效减小TS-1晶体在某一方向上 ( $b$ 方向)的尺寸(图10F), 改善反应物和产物的扩散性 能, 提高1-已烯环氧化TOF值到 $150 \mathrm{~h}^{-1}$.

生长抑制剂可以通过阻断硅源、钛源和 TS-1 纳米 晶体之间的缩合生长来抑制 TS- 1 铁硅分子篮的长大, 也可以通过调控硅源、钛源与TS-1纳米晶体特定位置 的配位作用来抑制 TS-1 钛硅分子篮的生长. 添加生长 抑制剂在有效调控TS-1 钛硅分子篮生长的同时, 也通 常会伴随晶间介孔的生成, 从而缩短分子扩散的路径 并改善分子扩散的空间位阻, 进而暴露大量的活性钛 位点, 改善烯烃环氧化反应性能.

\section{5 总结与展望}

本文针对烯烃高效环氧化反应从不同层次上简要 总结了精准设计与理性构筑高性能钛位点三大策略: 在原子尺度上设计钛位点特定的配位形式, 在界面上 调节钛位点的微反应环境, 以及在纳米颗粒中改善钛 位点的可接触性. 通常认为钛硅分子篮中的骨架钛物 种是烯烃环氧化的活性中心, 因此直接从原子尺度设 计钛原子的配位形式可以从反应源头有效改善烯烃环 氧化的性能. 此外, 在界面上调节反应活性中心骨架钛 物种周边的微反应环境(如酸碱性位点和硅羟基)将会 深刻影响环氧化产物的后续深度反应, 如脱附、开环 以及聚合等. 因此, 精准调控酸碱性位点和硅羟基的 空间分布和数目将会改善环氧化产物的反应行为. 受 制于TS-1 钛硅分子篮狭窄的微孔孔径, 烯烃相关反应 物和产物(尤其是大分子烯烃)的传质扩散受到极大抑 制. 为解决传质上的弊端, 构造多级孔道和合成纳米尺 度的TS-1能够有效改善分子的扩散传质, 大幅提高活 性骨架钛位点的可接触性, 进而提升烯烃环氧化的反 应性能.

尽管在过去的几十年中, TS-1钛硅分子笁在烯烃 高效环氧化上取得了令人瞩目的成就, 但仍然存在许 多挑战, 尤其是在精准合成钛物种的配位形式、调控 钛物种空间精确位置和采用廉价方法改善分子篎传质 性能等领域仍然具有挑战性. 原子级的钠配位化学仍然 是TS-1钛硅分子篮未来的核心热点研究内容, 本文凝练 的调控策略可以为钛物种的精确配位提供一定的理论 指导. 此外, 烯烃环氧化的真正活性中心仍然需要更加 
精密的原位实验表征和更准确的模拟计算来证明, 这对 表征技术和理论计算的迭代升级提出了巨大挑战. 我们
坚信, 钠硅分子篮正处在不断的发展和革新中, 这会进 一步促进“绿色烯烃环氧化”的工业化进程.

\section{参考文献}

1 Bao XH, Ding KL, He MY, Xie ZK. Sci Sin Chim, 2020, 50: 729-731 [包信和, 丁奎岭, 何鸣元, 谢在库. 中国科学: 化学, 2020, 7: 729-731]

2 Anastas P, Eghbali N. Chem Soc Rev, 2010, 39: 301-312

3 Sabatini MT, Boulton LT, Sneddon HF, Sheppard TD. Nat Catal, 2019, 2: 10-17

4 Poliakoff M, Licence P. Nature, 2007, 450: 810-812

5 Climent MJ, Corma A, Iborra S. Chem Rev, 2011, 111: 1072-1133

6 Jullesson D, David F, Pfleger B, Nielsen J. Biotechnol Adv, 2015, 33: 1395-1402

Zhou X, Yan H, Feng X, Zhao H, Liu Y, Chen X, Yang C. Ind Eng Chem Res, 2020, 59: 20086-20101

Mei XY. Petroleum Petrochem Today, 2007, 15: 34-37

Bregante DT, Flaherty DW. J Am Chem Soc, 2017, 139: 6888-6898

10 Jin K, Maalouf JH, Lazouski N, Corbin N, Yang D, Manthiram K. J Am Chem Soc, 2019, 141: 6413-6418

11 Bregante DT, Priyadarshini P, Flaherty DW. J Catal, 2017, 348: 75-89

12 Feng X, Yang J, Duan X, Cao Y, Chen B, Chen W, Lin D, Qian G, Chen D, Yang C, Zhou X. ACS Catal, 2018, 8: 7799-7808

13 Feng X, Song Z, Liu Y, Chen X, Jin X, Yan W, Yang C, Luo J, Zhou X, Chen D. ACS Catal, 2018, 8: 10649-10657

14 Lee WS, Cem Akatay M, Stach EA, Ribeiro FH, Nicholas Delgass W. J Catal, 2013, 308: 98-113

15 Lee WS, Cem Akatay M, Stach EA, Ribeiro FH, Nicholas Delgass W. J Catal, 2014, 313: 104-112

Lu X, Zhou WJ, Wu H, Liebens A, Wu P. Appl Catal A-Gen, 2016, 515: 51-59

Lin D, Zheng X, Feng X, Sheng N, Song Z, Liu Y, Chen X, Cai Z, Chen D, Yang C. Green Energy Environ, 2020, 5: 433-443

Yuan J, Song Z, Lin D, Feng X, Tuo Y, Zhou X, Yan H, Jin X, Liu Y, Chen X, Chen D, Yang C. ACS Appl Mater Interfaces, 2021, 13: 2613426142

19 Feng X, Chen D, Zhou XG. RSC Adv, 2016, 6: 44050-44056

20 Feng X, Duan X, Cheng H, Qian G, Chen D, Yuan W, Zhou X. J Catal, 2015, 325: 128-135

21 Feng X, Duan X, Qian G, Zhou X, Chen D, Yuan W. J Catal, 2014, 317: 99-104

Song Z, Feng X, Liu Y, Yang C, Zhou X. Prog Chem, 2016, 28: 1762-1773

Marimuthu A, Zhang J, Linic S. Science, 2013, 339: 1590-1593

Kim J, Chun J, Ryoo R. Chem Commun, 2015, 51: 13102-13105

Přech J, Kim J, Mazur M, Ryoo R, Čejka J. Adv Mater Interfaces, 2021, 8: 2001288

Kim SK, Reddy BM, Park SE. Ind Eng Chem Res, 2018, 57: 3567-3574

Wu G, Lin Z, Li L, Zhang L, Hong Y, Wang W, Chen C, Jiang Y, Yan X. Chem Eng J, 2017, 320: 1-10

Antonova NS, Carbó JJ, Kortz U, Kholdeeva OA, Poblet JM. J Am Chem Soc, 2010, 132: 7488-7497

Nie X, Ren X, Ji X, Chen Y, Janik MJ, Guo X, Song C. J Phys Chem B, 2019, 123: 7410-7423

Liu X, Yang C, Wang Y, Guo Y, Guo Y, Lu G. Chem Eng J, 2014, 243: 192-196

Shan Z, Lu Z, Wang L, Zhou C, Ren L, Zhang L, Meng X, Ma S, Xiao FS. ChemCatChem, 2010, 2: 407-412

Feng X, Lin D, Chen D, Yang C. Sci Bull, 2021, 66: 1945-1949

Serrano DP, Sanz R, Pizarro P, Moreno I, Medina S. Appl Catal B-Environ, 2014, 146: 35-42

Wang L, Dai J, Xu Y, Hong Y, Huang J, Sun D, Li Q. J Mater Chem A, 2020, 8: 4428-4436

Feng X, Duan X, Qian G, Zhou X, Chen D, Yuan W. Appl Catal B-Environ, 2014, 150-151: 396-401

Du M, Zhan G, Yang X, Wang H, Lin W, Zhou Y, Zhu J, Lin L, Huang J, Sun D, Jia L, Li Q. J Catal, 2011, 283: 192-201

Feng X, Liu Y, Li Y, Yang C, Zhang Z, Duan X, Zhou X, Chen D. AIChE J, 2016, 62: 3963-3972

Zuo Y, Liu M, Zhang T, Hong L, Guo X, Song C, Chen Y, Zhu P, Jaye C, Fischer D. RSC Adv, 2015, 5: 17897-17904

Xu W, Zhang T, Bai R, Zhang P, Yu J. J Mater Chem A, 2020, 8: 9677-9683 
40 Zuo Y, Liu M, Guo X. Coordination states and catalytic performance of Ti in titanium silicalite-1. In: Srivastva AN, Ed.. Stability and Applications of Coordination Compounds. London: IntechOpen, 2019

41 Langerame F, Salvi AM, Silletti M, Moretti G. Surf Interface Anal, 2008, 40: 695-699

42 Wei Y, Li G, Su R, Lu H, Guo H. Appl Catal A-Gen, 2019, 582: 117108

43 Tang B, Dai W, Sun X, Guan N, Li L, Hunger M. Green Chem, 2014, 16: 2281-2291 Lu T, Zou J, Zhan Y, Yang X, Wen Y, Wang X, Zhou L, Xu J. ACS Catal, 2018, 8: 1287-1296 2019, 11: 1673-1680

Li H, Xu B, Deng B, Yan X, Zheng Y. Catal Commun, 2014, 46: 224-227 

2132

90 Peregot G, Bellussi G, Corno C, Taramasso M, Buonomot F, Esposito A. Studies in Surface Science and Catalysis. Netherlands: Elsevier, 1986

91 Sudhaka Rreddy J, Kumar R. J Catal, 1991, 130: 440-446

92 Cundy CS, Forrest JO. Microporous Mesoporous Mater, 2004, 72: 67-80

93 Corma A, Camblor MA, Esteve P, Martinez A, Perezpariente J. J Catal, 1994, 145: 151-158 2220-2222

Song Z, Yuan J, Cai Z, Lin D, Feng X, Sheng N, Liu Y, Chen X, Jin X, Chen D, Yang C. Green Energy Environ, 2020, 5: 473-483

Su J, Xiong G, Zhou J, Liu W, Zhou D, Wang G, Wang X, Guo H. J Catal, 2012, 288: 1-7

Chen Y, Wu Y, Zhang Y, Long L, Tao L, Yang M, Tang N. J Mol Catal A-Chem, 2012, 352: 102-109

Peng P, Stosic D, Aitblal A, Vimont A, Bazin P, Liu XM, Yan ZF, Mintova S, Travert A. ACS Catal, 2020, 10: 6822-6830

Barbera K, Bonino F, Bordiga S, Janssens TVW, Beato P. J Catal, 2011, 280: 196-205

Akouche M, Gilson JP, Nesterenko N, Moldovan S, Chateigner D, Siblani HE, Minoux D, Dath JP, Valtchev V. Chem Mater, 2020, 32: $2123-$

Blasco T, Camblor MA, Corma A, Esteve P, Guil JM, Martínez A, Perdigón-Melón JA, Valencia S. J Phys Chem B, 1998, 102: 75-88

Serrano DP, Li HX, Davis ME. J Chem Soc Chem Commun, 1992, 745

Anand R, Shevade SS, Ahedi RK, Mirajkar SP, Rao BS. Catal Lett, 1999, 62: 209-213

Li C, Xiong G, Xin Q, Liu J, Ying P, Feng Z, Li J, Yang W, Wang Y, Wang G, Liu X, Lin M, Wang X, Min E. Angew Chem Int Ed, 1999, 38 :

Du S, Sun Q, Wang N, Chen X, Jia M, Yu J. J Mater Chem A, 2017, 5: 7992-7998

Lv G, Deng S, Yi Z, Zhang X, Wang F, Li H, Zhu Y. Chem Commun, 2019, 55: 4885-4888

Chen R, Liu C, Johnson NW, Zhang L, Mahendra S, Liu Y, Dong Y, Chen M. Chem Eng J, 2019, 371: 193-202

Shakeri M, Dehghanpour SB. Microporous Mesoporous Mater, 2020, 298: 110066

Wang X, Xiao C, Zheng P, Zhao Z, Alabsi MH, Shi Y, Gao D, Duan A, Huang KW, Xu C. J Catal, 2020, 384: 136-146

Gao X, Zhou Y, Gu J, Li L, Li Y. Microporous Mesoporous Mater, 2019, 275: 263-269

Jin Z, Wang L, Zuidema E, Mondal K, Zhang M, Zhang J, Wang C, Meng X, Yang H, Mesters C, Xiao FS. Science, 2020, 367: 193-197

Liu XF, Wang XD, Na P, Jiang HY, Lang L, Zhao HS, Zhang BQ. Chin Sci Bull, 2010, 55: 3131-3137

Moreno-Fernandez G, Perez-Ferreras S, Pascual L, Llorente I, Ibañez J, Rojo JM. Electrochim Acta, 2018, 268: 121-130

Tesfu-Zeru T, Sakthivel M, Drillet JF. Appl Catal B-Environ, 2017, 204: 173-184

Feliczak-Guzik A. Microporous Mesoporous Mater, 2018, 259: 33-45

Bai R, Song Y, Bai R, Yu J. Adv Mater Interfaces, 2021, 8: 2001095

Fang Y, Hu H. Catal Commun, 2007, 8: 817-820

Wang X, Li G, Wang W, Jin C, Chen Y. Microporous Mesoporous Mater, 2011, 142: 494-502

Ok DY, Jiang N, Prasetyanto EA, Jin H, Park SE. Microporous Mesoporous Mater, 2011, 141: 2-7

Xin H, Zhao J, Xu S, Li J, Zhang W, Guo X, Hensen EJM, Yang Q, Li C. J Phys Chem C, 2010, 114: 6553-6559

Schmidt I, Krogh A, Wienberg K, Carlsson A, Brorson M, Jacobsen C J. Chem Commun, 2000, 2157-2158

Lakiss L, Rivallan M, Goupil JM, El Fallah J, Mintova S. Catal Today, 2011, 168: 112-117

Kremer SPB, Kirschhock CEA, Aerts A, Villani K, Martens JA, Lebedev OI, Van Tendeloo G. Adv Mater, 2003, 15: 1705-1707

Stein A, Wilson BE, Rudisill SG. Chem Soc Rev, 2013, 42: 2763-2803

Wang Z, Kiesel ER, Stein A. J Mater Chem, 2008, 18: 2194-2200

Wu L, Li Y, Fu Z, Su BL. Natl Sci Rev, 2020, 7: 1667-1701

Yuan W, Yuan P, Liu D, Yu W, Laipan M, Deng L, Chen F. J Colloid Interface Sci, 2016, 462: 191-199

Chen C, Wang H, Han C, Deng J, Wang J, Li M, Tang M, Jin H, Wang Y. J Am Chem Soc, 2017, 139: 2657-2663

Du Q, Guo Y, Duan H, Li H, Chen Y, Liu H. Fuel, 2017, 188: 232-238

Serrano DP, Sanz R, Pizarro P, Moreno I, Shami S. Microporous Mesoporous Mater, 2014, 189: 71-82

Cheneviere Y, Chieux F, Caps V, Tuel A. J Catal, 2010, 269: 161-168 
125

126

127

128

129

130

131

132

133

134

135

136

137

138

139

140

141

142

143

144

145

146

147

Wang Z, Xu L, Jiang J, Liu Y, He M, Wu P. Microporous Mesoporous Mater, 2012, 156: 106-114

Wilde N, Pelz M, Gebhardt SG, Gläser R. Green Chem, 2015, 17: 3378-3389

Bai R, Sun Q, Song Y, Wang N, Zhang T, Wang F, Zou Y, Feng Z, Miao S, Yu J. J Mater Chem A, 2018, 6: 8757-8762

Chen LH, Li XY, Tian G, Li Y, Rooke JC, Zhu GS, Qiu SL, Yang XY, Su BL. Angew Chem Int Ed, 2011, 50: 11156-11161

Kärger J, Valiullin R. Chem Soc Rev, 2013, 42: 4172-4197

Schneider D, Mehlhorn D, Zeigermann P, Kärger J, Valiullin R. Chem Soc Rev, 2016, 45: 3439-3467

Dang Y, Yao Y, Liu Y, Wei B, Feng X, Chen X, Yang C. Chem Eng Sci, 2019, 204: 110-117

Jiao Y, Adedigba AL, He Q, Miedziak P, Brett G, Dummer NF, Perdjon M, Liu J, Hutchings GJ. Catal Sci Technol, 2018, 8: 2211-2217

Li J, Zhang F, Zong L, Wang X, Wei H. Catalysts, 2021, 11: 113

Du S, Chen X, Sun Q, Wang N, Jia M, Valtchev V, Yu J. Chem Commun, 2016, 52: 3580-3583

Zuo Y, Liu M, Ma M, Song C, Guo X. Ind Eng Chem Res, 2017, 56: 7462-7467

Qiu F, Wang X, Zhang X, Liu H, Liu S, Yeung KL. Chem Eng J, 2009, 147: 316-322

Li Y, Fan Q, Li Y, Feng X, Chai Y, Liu C. Appl Surf Sci, 2019, 483: 652-660

Song Z, Feng X, Sheng N, Lin D, Li Y, Liu Y, Chen X, Zhou X, Chen D, Yang C. Catal Today, 2018, 347: 102-109

Du Q, Guo Y, Wu P, Liu H, Chen Y. Microporous Mesoporous Mater, 2019, 275: 61-68

Ke X, Xu L, Zeng C, Zhang L, Xu N. Microporous Mesoporous Mater, 2007, 106: 68-75

Xu W, Dong J, Li J, Li J, Wu F. J Chem Soc Chem Commun, 1990, 755: 755-756

Matsukata M, Ogura M, Osaki T, Hari Prasad Rao PR, Nomura M, Kikuchi E. Top Catal, 1999, 9: 77-92

Zhou J, Hua Z, Cui X, Ye Z, Cui F, Shi J. Chem Commun, 2010, 46: 4994-4996

Sanz R, Serrano DP, Pizarro P, Moreno I. Chem Eng J, 2011, 171: 1428-1438

Li N, Wang M, You Q, Bi C, Chen H, Liu B, Sun M, Hao Q, Zhang J, Ma X. Catal Sci Technol, 2020, 10: 1323-1335

Shen X, Wang J, Liu M, Li M, Lu J. Catal Lett, 2019, 149: 2586-2596

Song X, Yang X, Zhang T, Zhang H, Zhang Q, Hu D, Chang X, Li Y, Chen Z, Jia M, Zhang P, Yu J. Inorg Chem, 2020, 59: 13201-13210 


\title{
Rationally constructed and regulated highly efficient Ti sites of titanium silicalite for olefin epoxidation
}

\author{
Dong $\operatorname{Lin}^{1}$, Xiang Feng ${ }^{1 *}$, Yibin Liu ${ }^{1}$, De Chen ${ }^{1,2}$, Chaohe Yang ${ }^{1}$ \\ ${ }^{1}$ State Key Laboratory of Heavy Oil Processing, China University of Petroleum, Qingdao 266580, China \\ ${ }^{2}$ Department of Chemical Engineering, Norwegian University of Science and Technology, Trondheim 7491, Norway \\ *Corresponding author (email: xiangfeng@upc.edu.cn)
}

\begin{abstract}
With the continuous promotion of high-end strategy for chemicals, the titanium silicalite-1 (TS-1) has attracted wide attention due to its unique oxidizing ability. The TS-1 has widespread applications in a series of reactions such as olefin epoxidation, cyclohexanone ammoxidation, and phenol hydroxylation. Therefore, the TS- 1 is labeled as a milestone in the field of molecular sieve catalysis. Rational design and precise construction of high-performance TS-1 are the key to catalyze olefin epoxidation. This article summarizes three strategies for constructing high-performance titanium sites in TS-1, which involves designing specific coordination forms of titanium sites at the atomic scale, adjusting the microenvironment of titanium sites on the interface, and improving the accessibility of titanium sites in the particle. Moreover, the action modes and reaction mechanisms of different strategies are elaborated in detail, and corresponding constructing methods and characterization means are summarized. Furthermore, the current challenges and future perspectives about high-performance titanium sites are further proposed.
\end{abstract}

Keywords: titanium silicalite, TS-1, olefin epoxidation, high-performance Ti sites, constructed strategy

doi: $10.1360 /$ SSC-2021-0182 